\title{
First Order Phase Transitions in Unbounded Spin Systems I: Construction of the Phase Diagram
}

\author{
Christian Borgs ${ }^{1}$ and Roger Waxler ${ }^{2}$ \\ 1 Theoretische Physik, ETH-Hönggerberg, CH-8093 Zürich, Switzerland \\ ${ }^{2}$ Department of Mathematics, University of British Columbia, Vancouver, BC, Canada
}

\begin{abstract}
The phase diagram and the corresponding infinite volume Gibbs states are constructed for a large class of continuous, unbounded spin models. Our construction relies on a partition of unity mapping our system onto an interacting contour system, a generalisation of Zahradnik's approach to Piragov Sinai theory to interacting contour systems, and a suitable mean field expansion around the minimas of the Hamiltonian.
\end{abstract}

\section{Introduction}

In this and a forthcoming paper, [1], we study continuous spin models with a single spin potential, $V$, whose minima are deep and widely separated. While such models have been extensively discussed in the literature if the minima of $V$ are related by a symmetry, [2-5], results concerning the general case without any symmetry were only obtained in recent years.

The first work in this direction is that of Imbrie, [6], who treated two dimensional Euclidean field theories in which the potential, $V$, is a polynomial in the fields. His idea was to use the cluster expansion of [2] to obtain a hard core interacting contoür system and to analyse this system using the techniques of Pirogov and Sinai, [7-9]. This turned out to be technically rather complicated because the resulting contour activities were not positive. Imbrie solved this problem by using a relatively involved resummation technique ${ }^{1}$.

An alternative approach would be that of Bricmont et al., [12], who mapped certain lattice (and continuum) gases with three or more particle species to an interacting contour model (with positive activities) and then studied this model using the methods of [7-9]. Their method has been extended to bounded continuous spin systems on the lattice, but it is not clear whether their techniques extend to the unbounded case as well.

${ }^{1}$ Recently, Borgs and Imbrie realised, [10], that this resummation can be avoided using the techniques of [11] rather than [7-9] since that approach may be generalised to contour models with complex activities. See $[10]$ for details 
Another approach is that of [13] (see also [14]) which starts from the very beginning with a generalised contour model and then analyses this model using the cluster expansion techniques of [15]. Their results are very similar to those described in the present work, with two differences. They only consider potentials, $V$, which have two minima, and they only consider the point in the phase diagram at which the two phases corresponding to these minima coexist. There is no doubt that their methods could be easily extended to include potentials with three or more minima, but extending their results to include points in the phase diagram away from coexistence would require substantial generalization.

Note also that none of the papers, [2-6], [12], or [13] treat the completeness question. That is, the question of whether all translation invariant infinite volume states are a convex combination of the particular states constructed, as expected.

The goal of the present work is to construct the phase diagram, and corresponding infinite volume states, of an unbounded spin model with single spin potential $V$ and nearest neighbor interaction $W$ (see Sect. 1 for precise definitions). We assume that $V$ has $N$ deep and widely separated minima of approximately the same depth, and that $V$ depends on parameters $\mu \in R^{N-1}$ in such a way that by varying $\mu$ one shifts the differences between these minima. We will need assumptions concerning the behaviour of $V$ and $W$ near the minima of $V$. We choose assumptions (A.1 of Sect. 1) which allow us to control the Gibbs factor, $e^{-H}$, for fields lying near a minimum of $V$, through a cluster expansion around the Gaussian approximation to $e^{-H}$. This part of our analysis is quite flexible. We could use, instead, any other single phase type expansion or, more generally, any other method (for example that of [16]) which allows us to establish strong clustering properties for the models in which all spins $R_{x}$ are constrained to lie near one of the minima of $V$.

Concerning the fluctuations between the various minima of $V$ we proceed as follows. Let $\chi_{q}\left(R_{x}\right)$ denote a characteristic function which forces the spin, $R_{x}$, to lie in a certain neighborhood, $U_{q}$, of the $q^{\text {th }}$ minimum and let $\chi_{0}=1-\sum_{q=1}^{N} \chi_{q}$. Given a volume, $\Lambda \subset Z^{v}$, we introduce a partition of unity

$$
1=\sum_{\omega: \Lambda \rightarrow\{0,1, \ldots, N\}} \chi_{\omega(x)}\left(R_{x}\right)
$$

and consider $\omega$ as a spin configuration of a discrete spin system with ground states $1, \ldots, N$. Defining contours as usual in the Pirogov-Sinai theory we obtain a representation of our model as a system of contours interacting via "fields" $R_{x} \in U_{q}$, for some $q \in\{1,2, \ldots, N\}$. (See Sect. 2 for details.)

At this point one is confronted with the problem lying at the core of the Pirogov-Sinai theory. One would like to obtain expansions by insisting that $\omega_{x}=q$ for $x$ near the boundary of $\Lambda$ and for some $q$. One then expects that if the $q^{\text {th }}$ minimum is stable (where the term stable must be defined) then the resulting contours have small activity. It is, however, not clear a priori which $q$ are stable. In addition, one encounters such boundary conditions for all $q$ at intermediate steps of the analysis. To deal with this problem we choose to use the technique developed in [11]. This technique is based on the introduction of auxiliary models, 
called truncated models, for which all contours have small activities and which turn out to differ from the actual models that we are interested in only for rather large contours. The truncated models may then be analysed by Mayer expansion techniques. If $h_{q}^{\prime}$ are the free energies of the truncated models one shows that the truncated and actual models with $q$-boundary conditions are identical if

$$
h_{q}^{\prime}=h=\min _{m} h_{m}^{\prime} .
$$

Using standard methods, we then construct, for $q$ satisfying (0.2), called the stable values for $q$, infinite volume states, $\langle\cdot\rangle_{q}$, which are small perturbations of the Gaussian approximation to $e^{-H}$ in $U_{q}$ (Theorem 1, Sect. 1). Making appropriate assumptions about the behaviour of $V$ as $\mu$ varies (corresponding to the "degeneracy removing condition" needed in the Pirogov-Sinai theory) we then construct the phase diagram. That is, we show that there is a point, $\mu_{0}$, for which all $q$ are stable, that there are lines, $\mu_{q}(t)$ starting at $\mu_{0}$, for which all $q^{\prime}$ except $q$ are stable, that there are surfaces, $\mu_{q_{1} q_{2}}(t, s)$, bounded by $\mu_{q_{1}}(t)$ and $\mu_{q_{2}}(t)$, for which all $q$ except $q_{1}$ and $q_{2}$ are stable, and so on (Theorem 2, Sect. 1 and Sect. 6). In a second paper, [1], we then show that all translation invariant infinite volume states obeying certain regularity conditions on the growth of the boundary condition are convex linear combinations of the $\langle\cdot\rangle_{q}$. This then completes the analysis.

The paper is organized as follows. In Sect. 1 we define the model and state the assumptions we use to prove Theorems 1 and 2. In Sect. 2 we use the partition of unity, (0.1), to rewrite the model as an interacting contour model. In Sect. 3 the truncated models are introduced and we prove that the truncated and actual models are identical if $h_{q}^{\prime}=h$. The cluster expansion we use to control the truncated models is introduced in Sect. 4, and its convergence is proven in Sect. 5. In Sect. 6 we discuss the construction of the phase diagram and in Sect. 7 we construct the infinite volume states, $\langle\cdot\rangle_{q}$.

\section{The model, Assumptions, Results}

We consider an unbounded spin model on the lattice $Z^{v}, v \geqq 2$, with spins $R_{x} \in R$,

$$
H\left(R_{\Lambda}\right)=\sum_{x} V\left(R_{x}\right)+\frac{1}{2} \sum_{\substack{x, y \in \Lambda \\|x-y|=1}} W\left(R_{x}, R_{y}\right),
$$

and partition function

$$
Z(\Lambda)=\int e^{-H\left(R_{\Lambda}\right)} d R_{\Lambda},
$$

defined for arbitrary finite subsets $\Lambda \subset Z^{v}$. As usual we write $|x-y|$ for the lattice distance $\sum_{i=1}^{v}\left|x_{i}-y_{i}\right| . V$ and $W$ take values in $R \cup\{+\infty\}$ and depend on the inverse temperature $\beta$, as well as parameters $\mu \in \mathscr{V}^{(0)}$, where $\mathscr{V}^{(0)}$ is an open neighborhood of $0 \in R^{N-1}, N \geqq 2$. For $\mu=0, V$ has $N$ absolute minima ${ }^{2}, R_{1}^{(0)}, \ldots, R_{N}^{(0)}$, which

\footnotetext{
${ }^{2}$ In fact we allow for a slightly more general situation, see assumptions A.1-A.3 below
} 
become deep and widely separated as $\beta$ grows (see assumption A.2 below). As a typical example one may consider a potential, $V(R)$, obtained by rescaling from a $\beta$-independent potential, $v(r)$, with non-degenerate minima $r_{1}, \ldots, r_{N}$ :

$$
V(R)=\beta v(R / \sqrt{\beta}),
$$

and similarly for $W$

$$
W\left(R, R^{\prime}\right)=\beta w\left(R / \sqrt{\beta}, R^{\prime} / \sqrt{\beta}\right)
$$

with, for example, $w\left(r, r^{\prime}\right)=\left|r-r^{\prime}\right|^{2}$.

We now will formulate several assumptions on $V$ and $W$, which will allow us to prove the theorems mentioned in the introduction. We start with

Assumption A.0 (A Priori Bounds on $V$ and $W$ ). For all $\beta<\infty, V$ and $W$ are bounded below, and $\exp (-V)$ is integrable. We will assume that $W$ is normalised in such a way that

$$
\min _{\boldsymbol{R}, \boldsymbol{R}^{\prime}} W\left(R, R^{\prime}\right)=0 .
$$

Note that $W\left(R_{x}, R_{y}\right)$ in $(1.1)$ can be replaced by $\frac{1}{2} W\left(R_{x}, R_{y}\right)+\frac{1}{2} W\left(R_{y}, R_{x}\right)$ without changing $H\left(R_{A}\right)$. We therefore assume without loss of generality that $W$ is symmetric, i.e.

$$
W\left(R, R^{\prime}\right)=W\left(R^{\prime}, R\right) .
$$

Clearly the above assumption ensures that the partition function, $Z(\Lambda)$, exists. The next two assumptions state that in certain disjoint, open, intervals $U_{q}=U_{q}(\beta) \subset R, q=1, \ldots, N$, the Gibbs factor, $e^{-H}$, is well approximated by appropriate Gaussians in $U_{q}$, whereas fluctuations away from $U_{q}$ are suppressed. More precisely we assume the existence of a $\mu$-independent, positive function $\tilde{\tau}(\beta) \rightarrow \infty$ as $\beta \rightarrow \infty$, such that for

$$
\begin{aligned}
& U_{q}=\left\{R \in R|| R-\left.R_{q}^{(0)}\right|^{2}<\tilde{\tau}(\beta)\right\}, \\
& U_{0}=R / \bigcup_{q=1}^{N} U_{q},
\end{aligned}
$$

the following assumptions are true $\left(q\right.$ and $q^{\prime}$ always denote integers between 1 and $N)$.

Assumption A.1 (Gaussian Fluctuations). There are constants $\eta, \eta^{\prime}, \eta^{\prime \prime}$ not depending on $\mu$ and $\beta$, as well as functions

$$
\begin{aligned}
m_{q}^{2} & =m_{q}^{2}(\beta, \mu) \geqq \eta>0, & & m_{q}^{2}<\eta^{\prime}<\infty \\
\kappa_{q} & =\kappa_{q}(\beta, \eta) \geqq 0, & & \kappa_{q}<\eta^{\prime \prime}<\infty \\
R_{q} & =R_{q}(\beta, \mu) \in U_{q}, & & e_{q}=e_{q}(\beta, \mu) \in R
\end{aligned}
$$

such that the following statements hold:

0) $U_{q} \cap U_{q^{\prime}}=\phi$ for $q \neq q^{\prime}$,

i) $V(R) \geqq(\eta / 2)\left(R-R_{q}\right)^{2}+e_{q}$ for $R \in U_{q}$, 
ii) there are functions $\xi(\beta) \rightarrow \infty$ as $\beta \rightarrow \infty, \delta(\beta) \rightarrow 0$ as $\beta \rightarrow \infty$ such that

$$
\begin{gathered}
\left|V(R)-e_{q}-\frac{m_{q}^{2}}{2}\right| R-\left.R_{q}\right|^{2} \mid \leqq \delta(\beta), \\
\quad\left|W\left(R, R^{\prime}\right)-\frac{\kappa_{q}}{2}\right| R-\left.R^{\prime}\right|^{2} \mid \leqq \delta(\beta), \\
\text { if } \quad\left|R-R_{q}\right| \leqq \xi(\beta), \quad\left|R^{\prime}-R_{q}\right| \leqq \xi(\beta),
\end{gathered}
$$

iii) $\left[R_{q}-\xi(\beta), R_{q}+\xi(\beta)\right] \subset U_{q}(\beta)$ and

$$
e^{-(\eta / 2) \xi^{2}(\beta)} \leqq \delta(\beta)
$$

iv) $R_{q}(\beta, 0)=R_{q}^{(0)}$

Assumption A.2 (Suppression of Non-Gaussian Fluctuations). There are constants $K_{1}>0, K_{2}<\infty, 0<\alpha<1$, independent of $\beta$ and $\mu$, such that the following statements are true:
i) $\min _{R \in U_{q^{\prime}} R^{\prime} \in U_{q^{\prime}}} W\left(R, R^{\prime}\right) \geqq K_{1} \tilde{\tau}(\beta)$ for all $q \neq q^{\prime}$,
ii) $\inf _{R \in R} V(R) \geqq e_{0} \equiv \min _{q} e_{q}$,
iii) $\int_{U_{0}} d R e^{-\alpha\left(V(R)-e_{0}\right)} \leqq K_{2} e^{-K_{1} \tilde{\tau}(\beta)}$

\section{Assumption A.3 (Regularity of $e_{q}$ ).}

i) For $\mu=0$ all $e_{q}$ 's are equal.

ii) There is a constant $K_{3}<\infty$, independent of $\mu$ and $\beta$, such that for all $\mu, \mu^{\prime} \in \mathscr{V}^{(0)}$,

$$
\left|e_{q}(\mu)-e_{q}\left(\mu^{\prime}\right)\right| \leqq K_{3} \tilde{\tau}(\beta)\left|\mu-\mu^{\prime}\right|,
$$

where $|\mu|$ denotes the $l_{2}$-norm of $\mu$.

Note that assumption A. 2 expresses the fact that fluctuations away from the absolute minimum of $V$ are suppressed. Since we want to construct the phase diagram in a neighborhood of the coexistence point, we also need bounds on the differences between the values $V\left(R_{q}\right)$ of the various minima. These follow from assumption A.3.

\section{Remarks.}

i) Assumption A.1 will be used to prove the convergence of a convenient mean field expansion for the "restricted partition function," $Z_{q}^{\text {res }}(\Lambda)$, obtained from $Z(\Lambda)$ by restricting all fields $R_{x}$ to $U_{q}$. We therefore can replace this assumption by any other assumption which guarantees the convergence of a suitable expansion for $Z_{q}^{\text {res }}(\Lambda)$.

ii) In the abstract context considered in this paper it might have been more natural to formulate assumptions A.2 and A.3 (as well as the following assumptions A.4 and A.5) using the free energy, $h_{q}^{\text {res }}$, of the restricted model instead of $e_{q}$. We choose $e_{q}$ because the resulting assumptions are easier to check for the models we want to consider in the future (for example the model considered in [17]). 
iii) In the case in which $V$ and $W$ are obtained by rescaling functions $v$ and $w$, where $r_{1}, \ldots, r_{N}$ are the minima of $v$ and $w\left(r, r^{\prime}\right) \geqq w(r, r)=0$, we set

$$
\begin{aligned}
\tilde{\tau}(\beta) & =O(\beta), \quad R_{q}=\sqrt{\beta} r_{q}, \\
e_{q} & =\beta v\left(r_{q}\right), \quad m_{q}^{2}=v^{\prime \prime}\left(r_{q}\right), \quad \kappa_{q}=\left.\frac{\partial^{2}}{\partial r^{2}} w\left(r, r^{\prime}\right)\right|_{r=r^{\prime}=r_{q}},
\end{aligned}
$$

$\xi(\beta)$ and $\delta(\beta)$ may, for example, be chosen as $O\left(\beta^{\lambda}\right), O\left(\beta^{3 \lambda-1 / 2}\right)$, respectively, with $0<\lambda<\frac{1}{6}$.

The goal of this paper is to construct infinite volume states, $\langle\cdot\rangle_{q}, q=1, \ldots, N$, which are (for $\beta$ large) small perturbations of the Gaussian with covariance $\left(m_{q}^{2}-\kappa_{q} \Delta\right)^{-1}$ and mean $R_{q}$ (see assumption A.1). The idea is to construct a cluster expansion for each choice of $q$, with "high temperature" polymers describing the fluctuations within $U_{q^{\prime}}$ and Peierls countours describing the fluctuations away from $U_{q}$. Using the above assumptions it will be possible to establish the convergence of these expansions for a certain subset, $S=S(\beta, \mu) \subset\{1, \ldots, N\}$, of "stable phase" $q \in S$.

In fact we will show, following an idea of Zahradnik, that for $\beta$ large enough and $\mu$ in a neighborhood of zero, $S$ is always nonempty and is characterised by a certain free energy condition

$$
S(\beta, \mu)=\left\{q \mid h_{q}^{\prime}(\beta, \mu)=\min _{\tilde{q}} h_{\tilde{q}}^{\prime}(\beta, \mu)\right\},
$$

where $h_{q}^{\prime}$ is the free energy of a certain auxiliary model (see Sect. 3).

We have assumed that for $\mu=0$ the $e_{q}(\beta, 0)$ are all equal independent of $q$. If the $e_{q}$ are well behaved as functions of $\mu$ then for each $q^{\prime} \in\{1,2, \ldots, N\}$ there will be a curve in $\mathscr{V}^{(0)}$, starting at $\mu=0$, along which all of the $e_{q}$ are equal for $q \neq q^{\prime}$ while $e_{q^{\prime}}$ differs from the rest. Similarly, for each distinct pair, $\left\{q_{1}, q_{2}\right\} \subset\{1,2, \ldots, N\}$ there will be a surface in $\mathscr{V}^{(0)}$ along which $e_{q}$ are equal only when $q \in\{1,2, \ldots, N\} \backslash\left\{q_{1}, q_{2}\right\}$, and so on until one finds hypersurfaces of dimension $N-1$ on which all $e_{q}$ differ from each other. One may ask to what extent this structure reflects the behaviour of $S(\beta, \mu)$ in the sense that there is some $\mu_{0} \in \mathscr{V}^{(0)}$ such that $S\left(\beta, \mu_{0}\right)=\{1,2, \ldots, N\}$, that for each $q$ there is a line starting at $\mu_{0}$ along which $S(\beta, \mu)=\{1,2, \ldots, N\} /\{q\}$, and so on. Phrased in the usual language of Piragov-Sinai theory we will show that, for $\beta$ large enough, the phase diagram is a continuous deformation of the zero temperature phase diagram in a neighborhood of $\mu=0$. To obtain this result, however, we will need some additional assumptions.

\section{Assumption A.4 (Degeneracy Removing Condition).}

(i) $e_{q}(\mu)$ is $C^{1}$ in $\mathscr{V}^{(0)}$ and let

$$
E_{q j}=\frac{d}{d \mu_{j}}\left(e_{q}-e_{n}\right)
$$

Then $E$ is invertible for all $\mu \in \mathscr{V}^{(0)}$.

(ii) $\left\|E^{-1}\right\| \leqq 1 / M(\beta)$ for all $\mu \in \mathscr{V}^{(0)}$, and $\mathscr{V}^{(0)}$ contains the sphere of radius $M(\beta)^{-\lambda}$ around 0 for some $\lambda<1$ and some constant $M(\beta) \rightarrow \infty$ as $\beta \rightarrow \infty$. 
Assumption A.5 (Regularity of $\partial \boldsymbol{V} / \partial \boldsymbol{\mu}$ and $\partial \boldsymbol{W} / \partial \boldsymbol{\mu})$. There are constants $K_{5}$ and $K_{6}(\beta)$, where $K_{5}$ is independent of $\mu$ and $\beta$ and $K_{6}$ satisfies

$$
\lim _{\beta \rightarrow \infty} \frac{K_{6}(\beta)}{M(\beta)}=0,
$$

and there is a constant $p>0$ such that

$$
\begin{aligned}
\left|\frac{d V(R)}{d \mu}\right| & \leqq K_{5}\left(\tilde{\tau}(\beta)+\left(V(R)-e_{0}\right)\right), \\
\left|\frac{d W\left(R, R^{\prime}\right)}{d \mu_{j}}\right| & \leqq K_{5}\left(\tilde{\tau}(\beta)+W\left(R, R^{\prime}\right)\right),
\end{aligned}
$$

and, for all $R \in U_{q}$, for each $q=1,2, \ldots, N$,

$$
\begin{aligned}
\left|\frac{d}{d \mu_{j}}\left(V(R)-e_{q}\right)\right| & \leqq K_{6}(\beta)\left|R-R_{q}\right|^{p}, \\
\left|\frac{d}{d \mu_{j}} W\left(R, R^{\prime}\right)\right| & \leqq K_{6}(\beta)\left(\left|R-R_{q}\right|^{p}+\left|R-R_{q}\right|^{p}\right) .
\end{aligned}
$$

Remark iv). For the rescaled model, a degeneracy removing condition on

$$
\tilde{E}_{q j}=\frac{d}{d \mu_{i}}\left(v\left(r_{q}\right)-v\left(r_{N}\right)\right)
$$

implies assumption A.4 with $M(\beta)=O(\beta)$. Assuming, for example, that $w$ does not depend on $\mu$, together with the condition

$$
\left|\frac{d}{d \mu_{j}} v(r)\right| \leqq \tilde{K}_{5}(1+v(r))
$$

and a Lipschitz condition on $d v / d \mu_{j}$ in an open neighborhood of $r_{q}$,

$$
\left|\frac{d}{d \mu_{j}}\left(v(r)-v\left(r^{\prime}\right)\right)\right| \leqq \tilde{K}_{6}\left|r-r^{\prime}\right|^{p},
$$

gives assumptions A.5 with

$$
K_{6}(\beta)=O\left(\beta^{1-p / 2}\right) .
$$

Using assumptions A.0 through A.5 we will prove the following two theorems. $A=A\left(R_{\text {supp } A}\right)$ always denotes a local observable, i.e. a complex valued function of finitely many variables $R_{x}, x \in \operatorname{supp} A,\|A\|^{(\alpha)}$ is the norm

$$
\|A\|^{(\alpha)}=\sup _{R_{\operatorname{supp} A}}\left|A\left(R_{\operatorname{supp} A}\right) \prod_{x \in \operatorname{supp} A} \exp \left(-(1-\alpha)\left(V\left(R_{x}\right)-e_{0}\right)\right)\right|,
$$

and $\langle\cdot\rangle_{q, \Lambda}$ denotes the expectation value

$$
\langle\cdot\rangle_{q, \Lambda}=\frac{1}{Z_{q}(\Lambda)} \int \cdot \prod_{x \in \partial \Lambda} \chi_{q}\left(R_{x}\right) e^{-H\left(R_{\Lambda}\right)} d R_{\Lambda^{\prime}}
$$


with $Z_{q}(\Lambda)$ chosen so that $\langle 1\rangle_{q, \Lambda}=1 . \chi_{q}(R)$ is the characteristic function of the event $R \in U_{q}$. The first theorem concerns the infinite volume limit of $\langle\cdot\rangle_{q, \Lambda}$.

Theorem 1. Assume that assumptions A.0 through A.3 are valid. Then there is a finite constant $\beta^{*}$ and an open neighborhood, $\mathscr{V}^{(1)} \subset \mathscr{V}^{(0)}$, of $\mu=0$ such that if $\beta \geqq \beta^{*}$ and $\mu \in \mathscr{V}^{(1)}$, then there is a non-empty subset, $S(\beta, \mu) \subset\{1,2, \ldots, N\}$, of stable boundary conditions, $q \in S(\beta, \mu)$, such that the limit $\langle A\rangle_{q}=\lim _{\Lambda \rightarrow \mathbb{Z}^{d}}\langle A\rangle_{q, \Lambda}$ exists for all $A$ with $\|A\|^{(\alpha)}<\infty .\langle A\rangle_{\mathrm{q}}$ is translation invariant and describes a small perturóation of the Gaussian with mean $R_{q}$ and covariance $\left(m_{q}^{2}-\kappa_{q} \Delta\right)^{-1}$ (see Theorems 7.3 and 7.4 for the precise bounds).

The second theorem is the construction of the phase diagram. It states that there is an open subset, $\mathscr{V}^{(2)} \subset \mathscr{V}^{(0)}$, in which $\left\{\mu \in \mathscr{V}^{(2)} \mid S(\beta, \mu)=\{1,2, \ldots, N\}\right\}$ is a point, $\mu_{0} \in \mathscr{V}^{(2)}$; that, for each $q \in\{1,2, \ldots, N\},\left\{\mu \in \mathscr{V}^{(2)} \mid S(\beta, \mu)=\{1,2, \ldots, N\} \backslash\{q\}\right\}=$ $\widetilde{\mathscr{V}}_{q}$ is an open curve one of whose end points is $\mu_{0}$; that, for each pair of distinct $q_{1}, q_{2} \in\{1,2, \ldots, N\},\left\{\mu \in \mathscr{V}^{(2)} \mid S(\beta, \mu)=\{1,2, \ldots, N\} \backslash\left\{q_{1}, q_{2}\right\}\right\}=\tilde{\mathscr{V}}_{q_{1}, q_{2}}$ is an open surface whose boundary in $\mathscr{V}^{(2)}$ is $\tilde{\mathscr{V}}_{q_{1}} \cup \widetilde{\mathscr{V}}_{q_{2}} \cup\left\{\mu_{0}\right\}$; and so on. More precisely

Theorem 2. Assuming assumptions A.0 through A.5 to be valid there is a finite constant $\beta^{*}$ and an open subset $\mathscr{V}^{(2)} \subset \mathscr{V}^{(1)} \subset \mathscr{V}^{(0)}$ such that for $\beta \geqq \beta^{*}$ and for all non-empty subsets $M \subset\{1,2, \ldots, N\}$ the set $\mathscr{V}_{M}=\left\{\mu \in \mathscr{V}^{(2)} \mid S(\beta, \mu) \subset M\right\}$ is a non-empty, continuous hypersurface of dimension $N-|M|$.

For further discussion of the relation between Theorem 2 and the phase diagram see Sect. 6.

\section{Dilute Partition Functions, Contours}

In this section we begin the contour analysis of our model. We will introduce certain dilute partition functions, $Z_{q}^{\mathrm{dil}}(\Lambda)$, for which the field $\phi_{x}$ is constrained to lie in $U_{q}$ near $\partial \Lambda$, and then rewrite $Z_{q}^{\mathrm{dil}}(\Lambda)$ as a sum over non-overlapping contours, interacting via a perturbed Gaussian.

We first fix some notation. For two points $x, y \in Z^{v}$ we usually use the maximum distance $\operatorname{dist}(x, y)=\max \left|x_{i}-y_{i}\right|$, except for the notation of a nearest neighbor pair $\langle x, y\rangle$, for which $1 \leqq i \leqq v$

$$
|x-y|=\sum_{i=1}^{v}\left|x_{i}-y_{i}\right|=1
$$

For $V, W \subset Z^{v}$ we define $\operatorname{dist}(V, W)$ as the minimum over all points $x \in V, y \in W$ of $\operatorname{dist}(x, y)$, and $\operatorname{diam} V=\max _{x, y \in V} \operatorname{dist}(x, y)$. We set $V^{c}=Z^{v} \backslash V, \partial V=\{x \in V \mid \operatorname{dist}(x, V) \leqq 1\}$ and say $V$ is connected if $\operatorname{dist}(W, V \backslash W) \leqq 1$ for any non-trivial subset $W \subset V$. We say $V$ is a volume without holes if $V^{c}$ is connected and call two sets $V, W$ adjacent or touching if $\operatorname{dist}(V, W) \leqq 1$.

Our partition functions will always be defined in sets $\Lambda$ of the form

$$
\Lambda=\bigcup_{x^{\prime} \in \Lambda^{\prime}} \square\left(x^{\prime}\right)
$$

where, for some odd integer $L>1$ to be chosen later, $\Lambda^{\prime}$ is a finite subset of $L Z^{v}$ 
and $\square\left(x^{\prime}\right)$ is an $L$-block centered at $x^{\prime}$,

$$
\square\left(x^{\prime}\right)=\left\{x \in Z^{v} \mid \operatorname{dist}\left(x, y^{\prime}\right) \leqq L / 2\right\} .
$$

We now introduce the partition of unity $1=\sum_{i=0}^{N} \chi_{i}(R)$, with

$$
\chi_{i}(R)=\left\{\begin{array}{lll}
0 & \text { if } & R \notin U_{i} \\
1 & \text { if } & R \in U_{i}
\end{array}\right.
$$

and rewrite $Z(\Lambda)$ as

$$
Z(\Lambda)=\sum_{\omega: \Lambda \rightarrow\{0, \ldots, N\}} \int \chi_{\omega}\left(R_{\Lambda}\right) e^{-H\left(R_{\Lambda}\right)} d R_{\Lambda},
$$

where we have introduced the notation $\chi_{\omega}\left(R_{\Lambda}\right)=\prod_{x \in \Lambda} \chi_{\omega(x)}\left(R_{x}\right)$.

The $q$-dilute partition function $Z_{q}^{\text {dil }}(\Lambda)$ is obtained from $Z(\Lambda)$ by restricting the sum over $\omega$ to a certain set $\Omega_{q}^{\mathrm{dil}}(\Lambda)$. For volumes $\Lambda$ without holes this is the set of configurations, $\omega$, for which $\omega(x)=q$ for all $x \in \Lambda$ with $\operatorname{dist}\left(x, \partial \Lambda^{c}\right) \leqq L+1$. For volumes $\Lambda$ with holes we will introduce additional constraints which forbid contours enclosing the holes of $\Lambda$ (this turns out to be convenient at several places, in particular in Sect. 3 and Sect. 7). We now define $\Omega_{q}^{\text {dil }}(\Lambda)$ for arbitrary volumes, $\Lambda$, of the form (2.1).

Given $\omega: \Lambda \rightarrow\{0,1, \ldots, n\}$ a block $\square\left(x^{\prime}\right) \subset \Lambda$ is called $q$-correct with respect to $\omega, q=1, \ldots, N$, if $\omega(x)=q \forall x \in \Lambda$ with $d\left(x, \square\left(x^{\prime}\right)\right) \leqq 1 . \square\left(x^{\prime}\right)$ is called incorrect with respect to $\omega$, if there is no $q \in\{1, \ldots, N\}$ such that $\square\left(x^{\prime}\right)$ is $q$-correct. The union of all blocks incorrect with respect to $\omega$ is denoted $B(\omega)$, and $\Omega_{q}^{\mathrm{dil}}(\Lambda)$ is defined as the set of all $\omega: \Lambda \rightarrow\{0,1, \ldots, N\}$, such that

i) all cubes $\square\left(x^{\prime}\right) \subset \Lambda$ which touch $\partial \Lambda^{c}$ are $q$-correct with respect to $\omega$ and

ii) all finite connect components of $(B(\omega))^{c}$ lie in $\Lambda$.

Letting

$$
H\left(R_{\Lambda} \mid R_{\Lambda^{\prime}}\right)=H\left(R_{\Lambda}\right)+\sum_{\substack{x \in \Lambda, y \in \Lambda^{\prime} \\|x-y|=1}} W\left(R_{x}, R_{y}\right),
$$

$\Lambda^{\prime} \subset \Lambda^{c}$, we define, for all finite $\Lambda$ of the form $(2.1), q \in\{1, \ldots, N\}$ and boundary conditions $R_{\Lambda^{\prime}}$ in $\left(U_{q}\right)^{\left|\Lambda^{\prime}\right|}$,

$$
\begin{gathered}
Z_{q}^{\mathrm{dil}}(\Lambda)=\sum_{\omega \in \Omega_{q}^{\mathrm{dil}}(\Lambda)} \int \chi_{\omega}\left(R_{\Lambda}\right) e^{-H\left(R_{\Lambda}\right)} d R_{\Lambda}, \\
Z_{q}^{\mathrm{dil}}\left(\Lambda \mid R_{\Lambda^{\prime}}\right)=\sum_{\omega \in \Omega_{q}^{\mathrm{dil}}(\Lambda)} \int \chi_{\omega}\left(R_{\Lambda}\right) e^{-H\left(R_{\Lambda} \mid R_{\Lambda^{\prime}}\right)} d R_{\Lambda^{\prime}}
\end{gathered}
$$

We also define the restricted partition functions

$$
\begin{aligned}
Z_{q}^{\mathrm{res}}(\Lambda) & =\int \chi_{q}\left(R_{\Lambda}\right) e^{-H\left(R_{\Lambda}\right)} d R_{\Lambda}, \\
Z_{q}^{\mathrm{res}}\left(\Lambda \mid R_{\Lambda^{\prime}}\right) & =\int \chi_{q}\left(R_{\Lambda}\right) e^{-H\left(R_{\Lambda^{\prime}} \mid R_{\Lambda^{\prime}}\right)} d R_{\Lambda} .
\end{aligned}
$$

We now wish to rewrite $Z_{q}^{\mathrm{dil}}(\Lambda)$ as a sum over contours. We proceed as follows. Given a function $\omega \in \Omega_{q}^{\text {dil }}(\Lambda)$ we decompose $B(\omega)$ into its connected components $V_{1}, \ldots, V_{k}$ and define $\omega_{i}$ to be the restrictions of $\omega$ to $V_{i}, i=1, \ldots, k$. The pairs 
$\gamma_{1}=\left(V_{1}, \omega_{1}\right), \ldots, \gamma_{k}=\left(V_{k}, \omega_{k}\right)$ are called the contours of $\omega$, and $V_{i}$ is called the support, $\operatorname{supp} \gamma_{i}$, of $\gamma_{i}$.

Fix a contour $\gamma=\left(\operatorname{supp} \gamma, \omega_{\gamma}\right)$ of $\omega$. Then, as may be verified from the definition of $q$-correct, $\omega$ is constant on the boundary, $\partial K$, of each connected component, $K$, of $(\operatorname{supp} \gamma)^{c}$. We let $\operatorname{Int}_{m} \gamma$ denote the union of all finite components, $K$, for which $\omega=m$ on $\partial K$ and write

$$
\operatorname{Int} \gamma=\bigcup_{m=1}^{n} \operatorname{Int}_{m} \gamma, \quad V(\gamma)=\operatorname{Int} \gamma \cup \operatorname{supp} \gamma, \quad \operatorname{Ext}(\gamma)=Z^{v} \backslash V(\gamma) .
$$

We also introduce the notions

$$
\partial \gamma=\partial \operatorname{supp} \gamma, \quad \partial_{m} \gamma=\partial\left(\operatorname{Int}_{m} \gamma\right)^{c}, \quad \partial_{e} \gamma=\partial V(\gamma)^{c} .
$$

Note that $\omega$ is constant on $\partial V(\gamma)$ (we say $\gamma$ is a $q$-contour if $\omega=q$ on $\partial V(\gamma)$ ) and that $\omega=m$ on $\partial_{m} \gamma$. A contour $\gamma$ is called an external contour of $\omega$ if $\operatorname{supp} \gamma \subset \operatorname{Ext}\left(\gamma^{\prime}\right)$ for all contours $\gamma^{\prime} \neq \gamma$ of $\omega$.

We also introduce the following notation: A pair $\gamma=\left(\operatorname{supp} \gamma, \omega_{\gamma}\right)$ is called a contour in $\Lambda$, if $\gamma$ is a contour of $\omega$ for some $q \in\{1, \ldots, n\}$ and some $\omega \in \Omega_{q}^{\text {dil }}(\Lambda)$ (note that by our definition of $\Omega_{q}^{\text {dil }}(\Lambda)$ this forbids contours which surround the holes of $\Lambda$ ). Two contours, $\gamma, \gamma^{\prime}$, are compatible if $\operatorname{dist}\left(\gamma, \gamma^{\prime}\right) \equiv \operatorname{dist}\left(\operatorname{supp} \gamma, \operatorname{supp}^{\prime}\right) \geqq 2$ and mutually external if $\operatorname{dist}\left(V(\gamma), V\left(\gamma^{\prime}\right)\right) \geqq 2$. Finally we let $\Omega_{q}^{\text {ext }}(\Lambda)$ denote the set of sets, $\left\{\gamma_{1}, \ldots, \gamma_{k}\right\}$, of mutually external $q$-contours in $\Lambda$.

Remark 2.1. Since supp $\gamma$ is a union of blocks centered in $L Z^{v}$, and any two such blocks, $\square\left(x^{\prime}\right), \square\left(x^{\prime \prime}\right)$, which do not touch have at least the distance $L+1$, any contour $\gamma$ in $\Lambda$ has at least distance $L+1$ from $\Lambda$, and two compatible contours have at least distance $L+1$ from one another.

The following procedure is standard in Pirogov-Sinai theory: Consider a configuration $\omega_{\Lambda} \in \Omega_{q}^{\text {dil }}(\Lambda)$ and its external contours $\gamma_{1}, \ldots, \gamma_{m}$. Resum over all $\omega_{\Lambda}$ with a given set, $C=\left\{\gamma_{1}, \ldots, \gamma_{m}\right\}$, of external contours. Introduce the notation

$$
e^{-\phi\left(y \mid R_{\gamma}\right)}=\chi_{\omega_{\gamma}}\left(R_{\gamma}\right) e^{-H\left(R_{\gamma}\right)}
$$

with $R_{\gamma}=R_{\text {supp } \gamma}$. Then $Z_{q}^{\text {dil }}(\Lambda)$ becomes

$$
\begin{aligned}
Z_{q}^{\mathrm{dil}}(\Lambda)= & \sum_{C \in \Omega_{q}^{\mathrm{ext}}(\Lambda)} \int d R_{\Lambda \backslash \operatorname{Int} C} e^{-H\left(R_{\mathrm{Ext}_{\Lambda} C} \backslash R_{\partial V(C)}\right)} \chi_{q}\left(R_{\mathrm{Ext}_{\Lambda} C}\right) \\
& \cdot \prod_{\gamma \in C} e^{-\phi\left(\gamma \mid R_{\gamma}\right)} \prod_{m=1}^{N} Z_{m}^{\mathrm{dil}}\left(\operatorname{Int}_{m} \gamma \mid R_{\partial_{m} \gamma}\right)
\end{aligned}
$$

where we have used the notation

$$
\text { Int } C=\bigcup_{\gamma \in C} \operatorname{Int} \gamma, \quad V(C)=\bigcup_{\gamma \in C} V(\gamma), \quad \text { and } \quad \operatorname{Ext}_{\Lambda} C=\Lambda \backslash V(C) .
$$

We now define the relative free energy, $F_{q}$, of a $q$-contour $\gamma$ by

$$
e^{-F_{q}\left(\gamma \mid R_{\partial \gamma}\right)}=\prod_{m=1}^{N} \frac{Z_{m}^{\mathrm{dil}}\left(\operatorname{Int}_{m} \gamma \mid R_{\partial_{m} \gamma}\right)}{Z_{q}^{\mathrm{dil}}\left(\operatorname{Int}_{m} \gamma\right)}
$$


and the contour functional $\psi_{q}(\cdot \cdot \cdot)$ by

$$
\begin{aligned}
\exp \left(-\psi_{q}\left(\gamma \mid R_{\partial_{e} \gamma}\right)\right) & \equiv \int d R_{\gamma} \chi_{\omega_{\gamma}}\left(R_{\gamma}\right) \exp \left(-H\left(R_{\gamma} \mid R_{\partial_{e} \gamma}\right)-F\left(\gamma \mid R_{\partial \gamma}\right)\right) \\
& =\int d R_{\gamma} \exp \left(-\phi\left(\gamma \mid R_{\gamma}\right)-F_{q}\left(\gamma \mid R_{\partial \gamma}\right)\right) \prod_{x \in \partial \gamma, y \in \partial_{e} \gamma|x-y|=1} \exp \left(-W\left(R_{x}, R_{y}\right)\right) .
\end{aligned}
$$

Dividing each term in (2.9) by $\prod_{\gamma \in C} Z_{q}\left(\operatorname{Int}_{\gamma}\right)$ and multiplying it back again we then obtain

$$
\begin{aligned}
Z_{q}^{\mathrm{dil}}(\Lambda)= & \sum_{C \in \Omega_{q}^{\mathrm{ext}}(\Lambda)} \int d R_{\mathrm{Ext}_{\Lambda} \mathrm{C}} \chi_{q}\left(R_{\mathrm{Ext}_{\Lambda} C}\right) \\
& \cdot \exp \left(-H\left(R_{\mathrm{Ext}_{\Lambda} C}\right)\right) \prod_{\gamma \in C} \exp \left(-\psi_{q}\left(\gamma \mid R_{e} \gamma\right)\right) Z_{q}^{\mathrm{dil}}\left(\mathrm{Int}_{\gamma}\right) .
\end{aligned}
$$

Equation (2.12) can be iterated and one obtains the following representation of $Z_{q}^{\text {dil }}(\Lambda)$ as a sum over sets, $C=\left\{\gamma_{1}, \ldots, \gamma_{k}\right\}$, of pair-wise compatible $q$-contours in $\Lambda$ (we write this sum as $\left.\sum_{C \text { in } \Lambda}^{q}\right)$

$$
Z_{q}^{\mathrm{dil}}(\Lambda)=\sum_{C \text { in } \Lambda}^{q} \int d R_{\Lambda \backslash C} \chi_{q}\left(R_{\Lambda \backslash C}\right) \exp \left(-H\left(R_{\Lambda \backslash C}\right)\right) \prod_{\gamma \in C} \exp \left(-\psi_{q}\left(\gamma \mid R_{\partial_{e} \gamma}\right)\right)
$$
where we have used the notation $\Lambda \backslash C=\Lambda \backslash \bigcup_{\gamma \in C} \operatorname{supp} \gamma$. We will sometimes also
write (2.13) as

$$
\sum_{C \text { in } \Lambda}^{q} \int d R_{\Lambda \backslash C} \chi_{q}\left(R_{\Lambda \backslash C}\right) \exp \left(-H\left(R_{\Lambda \backslash C}\right)\right) \exp \left(-\psi_{q}\left(C \mid R_{\partial_{e} C}\right)\right)
$$

where we have introduced the notation

$$
\psi_{q}\left(C \mid R_{\partial_{e} C}\right)=\sum_{\gamma \in C} \psi_{q}\left(\gamma \mid R_{\partial_{e} \gamma}\right) .
$$

Equation (2.13) is the desired representation of $Z_{q}^{\text {dil }}(\Lambda)$ as a sum over non-overlapping contours interacting via a perturbed Gaussian. The convergence of the corresponding Mayer series for $\log Z_{q}^{\text {dil }}(\Lambda)$ will be studied in Sects. 3-5.

\section{Stable and Unstable Contours, Truncated Contour Models}

In this section we begin the analysis of the convergence of the Mayer expansion for $\log Z_{q}^{\mathrm{dil}}(\Lambda)$. To motivate our strategy, assume for the moment that $Z_{q}^{\text {res }}$, defined in Eq. (2.5), can be written as the partition function of a dilute polymer model (this is in fact possible due to assumption A.1) and that $\psi_{q}$ obeys a bound of the form

$$
\left.\exp \left(-\psi_{q}\left(\gamma \mid R_{\partial_{e} \gamma}\right)\right) \leqq \exp \left(-\tau+e_{q}\right)|\operatorname{supp} \gamma|\right)
$$

for all $q$-contours, $\gamma$, and all boundary conditions $R_{\partial_{e} \gamma} \in U_{q}^{\left|\partial_{e} \gamma\right|}$. Then, for $\tau$ large enough, we can combine the contours in the sum (2.13) with the polymers of $Z_{q}^{\text {res }}$ to obtain a new polymer system which is again dilute. The resulting polymer expansion for $\log Z_{q}^{\text {dil }}(\Lambda)$ would be the desired convergent Mayer expansion.

While a bound of the form (3.1) can be established for the integral 
$\int \exp \left(-\phi\left(\gamma \mid R_{\gamma}\right)\right) d R_{\gamma}$, see Lemma 3.1 below, we cannot expect that (3.1) holds for $\psi_{q}(\cdot \cdot)$, at least not in general (otherwise one could show that the Gibbs states corresponding to $Z_{q}^{\text {dil }}$ are small perturbations of the corresponding Gaussian, for $\beta$ large and for all $q \in\{1, \ldots, N\}$, which is clearly false in general).

To overcome this difficulty we follow the strategy of Zahradnik [11] and introduce certain truncated contour functionals, $\psi_{q}^{\prime}$, for which a bound of the form (3.1) is true. In our context this is done by replacing $F_{q}\left(\gamma \mid R_{\partial \gamma}\right)$ in the definition of $\psi_{q}$ by $F_{q}^{\prime}\left(\gamma \mid R_{\partial \gamma}\right)=\max \left\{F_{q}\left(\gamma \mid R_{\partial \gamma}\right),-(2 \tau / 3)|\operatorname{supp} \gamma|\right\}$. The corresponding partition function, $Z_{q}^{\prime}$, can then be analysed by a convergent cluster expansion. In a final step one shows that $\psi_{q}$ and $\psi_{q}^{\prime}$ agree for those $q$, for which $h_{q}^{\prime}=h$, where $h_{q}^{\prime}$ is the free energy corresponding to $Z_{q}^{\prime}$ and $h=\min _{m} h_{m}^{\prime}$. Thus, if $h_{q}^{\prime}=\min _{m} h_{m}^{\prime}$, the cluster expansion for the truncated partition function, $Z_{q}^{\prime}$, is in fact a cluster expansion for $Z_{q}^{\text {dil }}$.

We begin by proving that the integral of $e^{-\phi}$ obeys a bound of the form (3.1). $K_{1}>0$ and $K_{3}<\infty$ are the constants from assumptions A.2 and A.3.

Lemma 3.1. There is a constant $c_{0}<\infty$ not depending on $L, \beta$, and $\mu$ such that for all

$$
\mu \in \mathscr{V}^{(1)}=\left\{\mu \in \mathscr{V}^{(0)}|| \mu \mid<\frac{K_{1}}{4 K_{3}}\left(2 L^{\nu}\right)^{-1}\right\}
$$

and all q-contours, $\gamma$, the following inequality holds:

$$
\int \exp \left(-\phi\left(\gamma \mid R_{\gamma}\right)\right) d R_{\gamma} \leqq \exp \left(-\left(\tau+e_{q}\right)|\operatorname{supp} \gamma|\right)
$$

with

$$
\tau=\tau(\beta, L) \equiv \frac{K_{1}}{2} \frac{\tilde{\tau}(\beta)}{(2 L)^{v}}-\log c_{0} .
$$

Proof. From assumption A.1 i),

$$
\begin{aligned}
\int e^{-V(R)} \chi_{m}(R) d R & \leqq e^{-e_{m}} \int \chi_{m}(R) \exp \left(-\frac{1}{2} \eta\left(R-R_{m}\right)^{2}\right) d R \\
& \leqq e^{-e_{m}} \int_{-\infty}^{\infty} \exp \left(-\frac{1}{2} \eta\left(R-R_{m}\right)^{2}\right) d R \leqq\left(\frac{2 \pi}{\eta}\right)^{1 / 2} e^{-e_{0}},
\end{aligned}
$$

where $m \neq 0$ and we recall that $e_{0}$ was defined as $\min e_{q}$. Given a $q$-contour, $\gamma$, we let $N(\Gamma)$ be the number of points, $x \in \operatorname{supp} \gamma$, for which $\omega_{\gamma}(x)=0$, and $\mathscr{L}(\gamma)$ be the number of links, $\langle x, y\rangle \subset \operatorname{supp} \gamma$, such that

$$
0 \neq \omega_{\gamma}(x) \neq \omega_{\gamma}(y) \neq 0 .
$$

Letting $c_{0}=\max \left\{K_{2},(2 \pi / \eta)^{1 / 2}\right\}$ we have from assumption A.2

$$
\begin{aligned}
\int \exp \left(-\phi\left(\gamma \mid R_{\gamma}\right)\right) d R_{\gamma} & =\int \exp \left(-H\left(R_{\gamma}\right)\right) \chi_{\omega_{\gamma}}\left(R_{\gamma}\right) d R_{\gamma} \\
& \leqq\left(c_{0} e^{-e_{0}}\right)^{|\operatorname{supp} \gamma|} \exp \left(-K_{1} \tilde{\tau}(N(\gamma)+\mathscr{L}(\gamma))\right) .
\end{aligned}
$$

Recall that by the definition of a contour each block $\square\left(x^{\prime}\right) \subset \operatorname{supp} \gamma$ must be incorrect. Therefore each block, $\square\left(x^{\prime}\right)$, must touch a link or point contributing to $\mathscr{L}(\gamma)$ or $N(\gamma)$ respectively. Since a link or point in $Z^{v}$ can touch at most $2^{v}$ distinct 
blocks we find that

$$
N(\gamma)+\mathscr{L}(\gamma) \geqq|\operatorname{supp} \gamma|(2 L)^{-\nu} .
$$

On the other hand, by assumption A.2,

$$
\left|e_{q}-e_{0}\right| \leqq 2 K_{3} \tilde{\tau}(\beta)|\mu| \leqq \frac{1}{2} K_{1} \frac{\tilde{\tau}(\beta)}{(2 L)^{v}},
$$

provided $\mu \in \mathscr{V}^{(1)}$. Combined with (3.5) and (3.6) this gives the bound (3.3).

We now define the truncated contour functional $\psi_{q}^{\prime}$. We will assume from now on that $\tau$ is the constant from Lemma 3.1 and that $\beta$ is chosen so large that $\tau>0$.

Definition 3.1. We define the truncated contour functional $\psi_{q}^{\prime}(\cdot \mid \cdot)$ by replacing $F_{q}\left(\gamma \mid R_{\partial \gamma}\right)$ in $(2.11)$ by

$$
F_{q}^{\prime}\left(\gamma \mid F_{\partial \gamma}\right)=\max \left\{F_{q}\left(\gamma \mid R_{\partial \gamma}\right),-\frac{2 \tau}{3}|\operatorname{supp} \gamma|\right\} .
$$

The truncated partition function $Z_{q}^{\prime}(\Lambda)$ is defined by the relation (2.13) with $\psi_{q}$ replaced by $\psi_{q}^{\prime}$.

With this definition $\psi_{q}^{\prime}$ obeys a bound of the form (3.1) (with $\tau$ replaced by $\tau / 3$ ). For $\beta$ large enough, $Z_{q}^{\prime}(\Lambda)$ can therefore be analysed by a convergent cluster expansion. This is done in Sects. 4 and 5, where we prove the following lemma $\left(\eta, \eta^{\prime}, \eta^{\prime \prime}\right.$ are the constants from assumption A.1).

Lemma 3.2. There are constants $L_{0}, c_{1}<\infty$ not depending on $L$ or $\beta$, as we all as $\beta$ independent constants $\tau_{1}=\tau_{1}(L), \beta_{1}=\beta_{1}(L)<\infty$ such that for $L \geqq L_{0}, \mu \in \mathscr{V}^{(1)}$, $\beta \geqq \beta_{1}$ and $\tau(\beta, L) \geqq \tau_{1}$, and for each $q \in\{1,2, \ldots, N\}$ there is a number $h_{q}^{\prime}$ which satisfies

i) $\left|h_{q}^{\prime}-e_{q}\right| \leqq c_{1}$,

ii) $\left|\log Z_{q}^{\prime}(\Lambda)+\right| \Lambda\left|h_{q}^{\prime}\right| \leqq c_{1}\left|\partial \Lambda^{c}\right|$.

Definition 3.2. Let $h_{q}^{\prime}$ be the free energy for the truncated contour model. We then define

$$
h=\min _{m} h_{m}^{\prime} \text { and } a_{q}=h_{q}^{\prime}-h .
$$

We call a $q$-contour, $\gamma$, stable if, $\forall m \in\{1, \ldots, N\}$,

and small if

$$
\left.\frac{Z_{m}\left(\operatorname{Int}_{m} \gamma\right)}{Z_{q}\left(\operatorname{Int}_{m} \gamma\right)} \leqq \exp (2 \tau / 3)\left|\partial_{m} \gamma\right|\right)
$$

$$
a_{q} \operatorname{diam} \gamma \leqq \tau / 3 .
$$

If $\gamma$ is not small we say it is large. Finally we define $q$ to be a stable phase if all $q$-contours are stable. We denote the set of stable phases by $S=S(\beta, \mu)$.

Remark 3.1. We have normalised $W$ in such a way that $W \geqq 0$. Therefore

$$
\exp \left(-F\left(\gamma \mid R_{\partial \gamma}\right)\right) \leqq \prod_{m} \frac{Z_{m}\left(\operatorname{Int}_{m} \gamma\right)}{Z_{q}\left(\operatorname{Int}_{m} \gamma\right)}
$$


On the other hand

$$
\sum_{m}\left|\partial_{m} \gamma\right| \leqq|\partial \gamma| \leqq|\operatorname{supp} \gamma|
$$

which implies that $\psi_{q}(\gamma \mid \cdot)=\psi_{q}^{\prime}(\gamma \mid \cdot)$ for stable contours $\gamma$. In particular $Z_{q}^{\prime}(\Lambda)=Z_{q}^{\mathrm{dil}}(\Lambda)$ if $q$ is a stable phase. It is, however, not a priori clear which are the stable phases, and whether there is at least one stable phase.

The following theorem implies that the set $S$ of stable phases is in fact not empty, and that a stable phase can be characterized by the condition $a_{q}=0$.

Theorem 3.3. Assume that $L$ has been chosen according to Lemma 3.2. Then there is a constant $\beta_{2}=\beta_{2}(L) \geqq \beta_{1}$, such that for $\beta \geqq \beta_{2}$ and $\mu \in \mathscr{V}^{(1)}$ the following statements hold:

i) $Z_{q}^{\mathrm{dil}}(\Lambda) \geqq \exp \left\{-h_{q}^{\prime}|\Lambda|-c_{1}\left|\partial \Lambda^{c}\right|\right\}$,

ii) $Z_{q}^{\mathrm{dil}}(\Lambda) \leqq \exp \left\{-h|\Lambda|+\left(c_{1}+1\right)\left|\partial \Lambda^{c}\right|\right\}$,

iii) If $\gamma$ is a small contour, then $\gamma$ is stable.

Here $\Lambda$ is an arbitrary finite volume of the form $(2.1)$ and $\beta_{1}, c_{1}$ are the constants of Lemma 3.2.

Remark 3.2. The constant $\beta_{2}$ is chosen in such a way that $\tau=\tau(\beta, L) \geqq \tau_{1}$ for $\beta \geqq \beta_{2}$. In addition we will need, in the course of the proof, the additional constraints

$$
\begin{aligned}
2 c_{1}+1 & \leqq \tau / 3, \\
\tau-3 c_{1}-1 & \geqq \tau_{2},
\end{aligned}
$$

where $\tau_{2}$ is a constant depending only on an $N$ and $v$.

Remark 3.3. Obviously Theorem 3.3 iii) implies that all phases $q$ with $a_{q}=0$ are stable. If, on the other hand, $q$ is a stable phase, then all $q$-contours are stable and

$$
\left.Z_{q}^{\prime}(\Lambda)=Z_{q}^{\mathrm{dil}}(\Lambda) \geqq Z_{m}^{\mathrm{dil}}(\Lambda) \exp (-2 \tau / 3)\left|\partial \Lambda^{c}\right|\right)
$$

for all $\Lambda$ and all $m$ by (3.10) (given $\Lambda$ and $m$, one just has to choose a $q$-contour $\gamma$ with $\operatorname{Int}_{m} \gamma=\Lambda$, which is always possible). Combined with Lemma 3.2 and Theorem 3.3 i) the above bound shows that $h_{q}^{\prime} \leqq h_{m}^{\prime}$ for all $m$, and hence $a_{q}=0$. So Theorem 3.3 implies in fact that $q$ is stable if and only if $a_{q}=0$.

Proof of Theorem 3.3 i). The statement follows immediately from Lemma 3.2 and the fact that $\psi^{\prime}\left(\gamma \mid R_{\partial_{e} \gamma}\right) \geqq \psi\left(\gamma \mid R_{\partial_{e} \gamma}\right)$.

Proof of Theorem 3.3 ii) and iii). The proof will be done by induction. We define the level of a contour $\gamma$ to be the maximal $n$ such that there are contours $\gamma_{1}, \ldots, \gamma_{n}=\gamma$, with supp $\gamma_{i} \subset \operatorname{Int} \gamma_{i+1}, \operatorname{dist}\left(V\left(\gamma_{i}\right), \gamma_{i+1}\right)>2$ and the level of $\Lambda$ as the maximal level of a contour in $\Lambda$.

Proof of ii) for level $\Lambda=0$. Trivial, because in this case $Z_{q}^{\mathrm{dil}}(\Lambda)=Z_{q}^{\mathrm{res}}(\Lambda)=Z_{q}^{\prime}(\Lambda)$.

Proof of iii) for level $\gamma=n$. Int $\operatorname{In}_{m}$ is a volume of level $\leqq n-1$. Therefore we can use the inductive assumption to bound

$$
\frac{Z_{m}^{\text {dil }}\left(\operatorname{Int}_{m} \gamma\right)}{Z_{q}^{\text {dil }}\left(\operatorname{Int}_{m} \gamma\right)} \leqq \exp \left(a_{q}\left|\operatorname{Int}_{m} \gamma\right|+\left(2 c_{1}+1\right)\left|\partial_{m} \gamma\right|\right) \text {. }
$$


Bounding $\left|\operatorname{Int}_{m} \gamma\right|$ by

$$
\left|\partial_{m} \gamma\right| \operatorname{diam}_{\operatorname{Int}} \gamma \leqq\left|\partial_{m} \gamma\right| \operatorname{diam} \gamma,
$$

and using the definition of a small contour we obtain the upper bound

$$
\exp \left(\left(2 c_{1}+1+\tau / 3\right)\left|\partial_{m} \gamma\right|\right)
$$

which shows that $\gamma$ is stable if

$$
\left.2 c_{1}+1 \leqq \tau / 3 \quad \text { (this is }(3.14 \mathrm{a})\right) \text {. }
$$

Proof of ii) for level $\Lambda=n$. We use the relation (2.9). Fix, for a moment, the set $C_{1}$ of large external contours in $C$. To specify $C$ completely, one has to specify, in addition to $C_{1}$, a set $C_{2}$ of mutually external, small contours in $\Lambda \backslash V\left(C_{1}\right)=$ Ext ${ }_{\Lambda}\left(C_{1}\right)$. Using the fact that the volume $\operatorname{Ext}_{\Lambda} C$ appearing in (2.9) can be rewritten as $\operatorname{Ext}_{\Lambda \backslash V\left(C_{1}\right)} C_{2}$ we can resum the small contours in $\Lambda \backslash V\left(C_{1}\right)$ to obtain the relation

$$
\begin{aligned}
Z_{q}^{\mathrm{dil}}(\Lambda)= & \sum_{C_{1}}^{\prime} \int Z_{q}^{\text {small }}\left(\Lambda \backslash V\left(C_{1}\right) \mid R_{\partial V\left(C_{1}\right)}\right) \\
& \cdot \prod_{\gamma \in C_{1}}\left[\exp \left(-\phi\left(\gamma \mid R_{\gamma}\right)\right) \prod_{m=1}^{N} Z_{m}^{\mathrm{dil}}\left(\operatorname{Int}_{m} \gamma \mid R_{\partial_{m} \gamma}\right) d R_{\gamma}\right],
\end{aligned}
$$

where $Z_{q}^{\text {small }}$ is obtained from $Z_{q}^{\text {dil }}$ by restricting the sum over $\omega$ in (2.4) to a sum over configurations $\omega$ which contain only small external contours, and $\sum^{\prime}$ is sum over sets of mutually external, large contours. We now bound

$$
\begin{gathered}
Z_{q}^{\text {small }}\left(\Lambda \backslash V\left(C_{1}\right) \mid R_{\partial V\left(C_{1}\right)}\right) \leqq Z_{q}^{\text {small }}\left(\Lambda^{\prime} V\left(C_{1}\right)\right), \\
Z_{m}^{\text {dil }}\left(\operatorname{Int}_{m} \gamma \mid R_{\partial_{\partial^{\gamma}}}\right) \leqq Z_{m}^{\text {dil }}\left(\operatorname{Int}_{m} \gamma\right),
\end{gathered}
$$

and use the fact that all the contours appearing in the representation (2.13) for $Z_{q}^{\text {small }}\left(\Lambda / V\left(C_{1}\right)\right)$ are stable due to the inductive assumption iii). Therefore $Z_{q}^{\text {small }}\left(\Lambda / V\left(C_{1}\right)\right) \leqq Z_{q}^{\prime}\left(\Lambda / V\left(C_{1}\right)\right)$ which can be bounded using Lemma 3.2. Using the inductive assumption ii) for $Z_{m}^{\mathrm{dil}}\left(\operatorname{Int}_{m} \gamma\right)$ and Lemma 3.1 for $\int \exp \left(-\phi\left(\gamma \mid R_{\gamma}\right)\right) d R_{\gamma}$ we get the bound

$$
\begin{aligned}
& Z_{q}^{\mathrm{dil}}(\Lambda) \leqq \sum_{C}^{\prime} \exp \left(-h_{q}^{\prime}|\Lambda \backslash V(C)|+c_{1}\left|\partial \Lambda^{c}\right|+c_{1}|\partial V(C)|\right) \\
& \prod_{\gamma \in C} \exp \left(-\left(\tau+e_{q}\right)|\operatorname{supp} \gamma|\right) \prod_{m} \exp \left(-h\left|\operatorname{Int}_{m} \gamma\right|+\left(1+c_{1}\right)\left|\partial_{m} \gamma\right|\right) .
\end{aligned}
$$

We now extract a factor $\exp (-h|\Lambda|)$, bound

$$
\left.\exp \left(-e_{q}|\operatorname{supp} \gamma|\right) \leqq \exp \left(-h_{q}^{\prime}-c_{1}\right)|\operatorname{supp} \gamma|\right),
$$

and use (3.13) to obtain the inequality

$Z_{q}^{\mathrm{dil}}(\Lambda) \leqq \exp \left(-h|\Lambda|+c_{1}\left|\partial \Lambda^{c}\right|\right) \sum_{C}^{\prime} \exp \left(-a_{q}|\Lambda \backslash \operatorname{Int} C|\right) \prod_{\gamma \in C} \exp \left(-\left(\tau-3 c_{1}-1\right)|\operatorname{supp} \gamma|\right)$.

To complete the proof, we need the following technical lemma, proven in [11]. For the convenience of the reader we give the proof of this lemma at the end of this section. 
Lemma 3.4 ([11]). Consider a contour functional $\tilde{K}: \gamma \mapsto \tilde{K}(\gamma) \geqq 0$ and let $\tilde{Z}$ be the partition function

$$
\tilde{Z}(\Lambda)=\sum_{C \text { in }}^{q} \prod_{\Lambda \in C} \tilde{K}(\gamma) e^{|\gamma|}
$$

Assume that $K(\gamma) \leqq \tilde{\varepsilon}^{|\gamma|}$. Then there is a constant $\tau_{2}$ depending only on $v$ and $N$, such that for $\tilde{\varepsilon} \leqq e^{-\tau_{2}}$ the following statement is true. Let $\tilde{s}$ be the free energy corresponding to $\tilde{Z}$. Then, for all $\tilde{a} \geqq-\tilde{s}$,

$$
\sum_{C}^{\prime} \exp (-\tilde{a}|\Lambda \backslash \operatorname{Int} C|) \prod_{\gamma \in C} \tilde{K}(\gamma) \leqq e^{\left|\partial \Lambda^{c}\right|},
$$

where the sum goes over sets of mutually external q-contours in $\Lambda$.

To apply this lemma we put $\tilde{a}=a_{q}$,

$$
\left.\tilde{\varepsilon}=\exp \left(-\gamma-3 c_{1}-1\right)\right)
$$

and

$$
\tilde{K}(\gamma)= \begin{cases}\tilde{\varepsilon}^{|\gamma|} & \text { if } \gamma \text { is large } \\ 0 & \text { if } \gamma \text { is small }\end{cases}
$$

Using the fact that $\tilde{Z}$ contains only contours $\gamma$ for which

$$
|\sup \gamma| \geqq\left(\tau / 3 a_{q}\right)
$$

(since $\operatorname{diam} \gamma \leqq|\operatorname{supp} \gamma|$, because $\operatorname{supp} \gamma$ is connected) one easily finds that

$$
-\tilde{s} \leqq O\left(\tilde{\varepsilon}^{\left(\tau / 3 a_{q}\right)}\right) \leqq a_{q}
$$

if $\tau$ is chosen large enough. We therefore can apply the lemma provided

$$
\tau-3 c_{1}-1 \geqq \tau_{2}
$$

(this is the restriction $3.14 \mathrm{~b}$ ).

We close this section with a proof of Lemma 3.4.

Proof of Lemma 3.4. $\tilde{Z}$ is the partition function of a polymer with activities $K^{*}(\gamma)=\tilde{K}(\gamma) e^{|\gamma|}$. For $\tilde{\varepsilon}$ small enough (depending only on $N$ and $v$ ) $\tilde{Z}$ can be controlled by a convergent expansion and

$$
|\log \tilde{Z}(W)+\tilde{s}| W|| \leqq O(\tilde{\varepsilon})\left|\partial W^{c}\right| \leqq\left|\partial W^{c}\right| .
$$

Putting $W=$ Int $C$ and using (3.13) together with the assumption $\tilde{a} \geqq-\tilde{s}$ we get

$$
\begin{aligned}
\sum_{C}^{\prime} \exp (-\tilde{a}|\Lambda \backslash \operatorname{Int} C|) \prod_{\gamma \in C} \tilde{K}(\gamma) & \leqq e^{\tilde{s}|\Lambda|} \sum_{C}^{\prime} \prod_{\gamma \in C} \tilde{K}(\gamma) e^{|\gamma|} \tilde{Z}(\operatorname{Int} \gamma) \\
& =e^{\tilde{s}|\Lambda|} \tilde{Z}(\Lambda) \leqq e^{\left|\partial \Lambda^{c}\right|}
\end{aligned}
$$

\section{Polymer Expansion for the Truncated Model}

In Sects. 4 and 5 we prove Lemma 3.2 by a convenient cluster expansion. In principle we could use the Glimm-Jaffe cluster expansion in the form of [18] to obtain a representation of $Z_{q}^{\text {res }}$ as a partition function of a hard core interacting 
polymer system and combine it with the contour expansion (2.13). This approach has however the disadvantage that it needs $C^{\infty}$-differentiability of $V$ and $W$ near $R_{q}$, which is not natural for the lattice models considered here.

Instead we use a slightly different approach, which, after expanding in the perturbing potentials

$$
\begin{gathered}
V_{q}(\phi) \equiv V\left(R_{q}+\phi\right)-\frac{m_{q}^{2}}{2} \phi^{2}-e_{q}, \\
W_{q}\left(\phi, \phi^{\prime}\right) \equiv W\left(R_{q}+\phi, R_{q}+\phi^{\prime}\right)-\frac{\kappa_{q}}{2}\left(\phi-\phi^{\prime}\right)^{2},
\end{gathered}
$$

uses interpolation parameters (s-parameters) only in regions where no factors of $V_{q}, W_{q}$ or $e^{-\psi_{q}^{\prime}}$ arise. Since we decouple these regions using non-zero Dirichlet data on their boundaries, we do not generate field derivatives of $V_{q}$ or $W_{q}$, and therefore don't need differentiability assumptions on $V_{q}$ or $W_{q}$. The convergence of this expression is established in Sect. 5.

We recall the definition of the truncated partition function $(\Lambda \backslash C$ is defined as $\left.\Lambda \backslash \bigcup_{\gamma \in C} \operatorname{supp} \gamma\right)$

$$
Z_{q}^{\prime}(\Lambda)=\sum_{C} \int d R_{\Lambda \backslash C} \chi_{q}\left(R_{\Lambda \backslash C}\right) \exp \left(-H\left(R_{\Lambda \backslash C}\right)\right) \prod_{\gamma \in C} \exp \left(-\psi_{q}^{\prime}\left(\gamma \mid R_{\partial_{e} \gamma}\right)\right),
$$

and rewrite, with $\phi_{x}=R_{x}-R_{q}$, where $R_{q}$ is the minimum of the quadratic approximation of $H$ in $U_{q}$,

$$
H\left(R_{\Lambda \backslash C}\right)=e_{q}|\Lambda \backslash \operatorname{supp} C|+H_{q}^{(0)}\left(\phi_{\Lambda \backslash C}\right)+\frac{1}{2} \sum_{x, y \in \Lambda \backslash C} W_{q}\left(\phi_{x}, \phi_{y}\right)+\sum_{x \in \Lambda \backslash C} V_{q}\left(\phi_{x}\right),
$$

where $V_{q}, W_{q}$ are defined in (4.1) and

$$
H_{q}^{(0)}\left(\phi_{\Omega}\right)=\frac{\kappa_{q}}{4} \sum_{\substack{x, y \in \Omega \\|x-y|=1}}\left(\phi_{x}-\phi_{y}\right)^{2}+\frac{m_{q}^{2}}{2} \sum_{x \in \Omega} \phi_{x}^{2} .
$$

We now expand in $V_{q}$ and $W_{q}$. More precisely, we define

$$
\begin{aligned}
F_{x y}\left(\phi_{x}, \phi_{y}\right) & =\exp \left(-W_{q}\left(\phi_{x}, \phi_{y}\right)\right)-1, \\
F_{x}\left(\phi_{x}\right) & =\chi_{q}\left(\phi_{x}+R_{q}\right) \exp \left(-V_{q}\left(\phi_{x}\right)\right)-1
\end{aligned}
$$

and, with the convention $F_{\varnothing}=1$,

$$
F_{X}\left(\phi_{X}\right)=\sum_{\substack{X_{1}, B, s_{1} \\ X=X_{1} \cup X(B)}} \prod_{x \in X_{1}} F_{x}\left(\phi_{x}\right) \prod_{\langle x, y\rangle \in B} F_{x y}\left(\phi_{x}, \phi_{y}\right),
$$

where $B$ denotes a set of nearest neighbor pairs and $X(B)$ is the corresponding set of points. Then

$$
\begin{aligned}
Z_{q}^{\prime}(\Lambda)= & e^{-e_{q}|\Lambda|} \sum_{C} \sum_{X \subset \Lambda \backslash C} \int d \phi_{\Lambda \backslash C} \exp \left(-H^{(0)}\left(\phi_{\Lambda \backslash C}\right)\right) \\
& \cdot F_{X}\left(\phi_{X}\right) \prod_{\gamma \in C} \exp \left(e_{q}|\operatorname{supp} \gamma|-\psi_{q}^{\prime}\left(\gamma \mid R_{q}+\phi_{\partial_{e} \gamma}\right)\right) .
\end{aligned}
$$


With the notation

$$
\begin{aligned}
Z_{q}^{(0)}\left(\Omega \mid \phi_{\Omega^{\prime}}\right) & =\int d \phi_{\Omega} \exp \left(-H_{q}^{(0)}\left(\phi_{\Omega} \mid \phi_{\Omega^{\prime}}\right)\right), \\
H_{q}^{(0)}\left(\phi_{\Omega} \mid \phi_{\Omega^{\prime}}\right) & =H_{q}^{(0)}\left(\phi_{\Omega}\right)+\frac{\kappa_{q}}{2} \sum_{\substack{x \in \Omega, y \in \Omega^{\prime} \\
|x-y|=1}}\left|\phi_{x}-\phi_{y}\right|^{2},
\end{aligned}
$$

this can be rewritten as

$$
\begin{aligned}
Z_{q}^{\prime}(\Lambda)= & e^{-e_{q}|\Lambda|} Z_{q}^{(0)}(\Lambda) \sum_{C} \sum_{X \subset \Lambda \backslash C} \int d \phi_{X \cup \partial_{e} C} \exp \left(-H_{q}^{(0)}\left(\phi_{X \cup \partial_{e} C}\right)\right) \\
& \cdot \prod_{\gamma \in C} \exp \left(-\psi_{q}^{\prime}\left(\gamma \mid R_{q}+\phi_{\partial_{e} \gamma}\right)+e_{q}|\operatorname{supp} \gamma|\right) F_{X}\left(\phi_{X}\right) \frac{Z_{q}^{(0)}\left(\Lambda_{1} \mid \phi_{\partial_{e} C \cup \partial X}\right)}{Z_{q}^{(0)}(\Lambda)},
\end{aligned}
$$

where $\Lambda_{1}=\Lambda \backslash\left(X \cup \operatorname{supp} C \cup \partial_{e} C\right)$. In order to decouple different connected components of $X \cup \operatorname{supp} C \cup \partial_{e} C$ we now choose a convenient expansion for the ratio of the Gaussian partition functions in Eq. (4.7). The expansion we choose is essentially that used in [18].

We note that there is a characteristic length, $l_{q}$, the correlation length of the gaussian, Eq. (4.3), over which points in the lattice are strongly correlated. It is this fact which motivated our introduction of the coarse lattice, $L Z^{v}$. Choosing $L>l_{q}$ we treat the couplings across faces of $L$-blocks as perturbations, but only for those $L$-blocks far enough from $\partial X \cup \partial_{e} C$ to sufficiently moderate the effects of the conditioning. Thus, given $X$ and $C$, define $\bar{X}$ to be the smallest union of $L$-blocks such that $X \cup \partial_{e} C \subset \bar{X} \subset \Lambda \backslash \operatorname{supp} C$ and $\operatorname{dist}(\Lambda \backslash(\bar{X} \cup \operatorname{supp} C)$, $\left.\partial X \cup \partial_{e} C\right)>r_{0}$, where $r_{0} \gg l_{q}$ will be fixed, along with $L$, in the next section. Given two nearest neighbor points, $x^{\prime}, y^{\prime}$, in $L Z^{v}$ define the face separating the adjacent $L$-blocks $x^{\prime}$ and $y^{\prime}$ to be the set of the $L^{v-1}$ nearest neighbor pairs $\{x, y\} \subset Z^{v}$ such that $\{x, y\} \cap \square\left(x^{\prime}\right) \neq \varnothing$ and $\{x, y\} \cap \square\left(y^{\prime}\right) \neq \varnothing$. Given a union of $L$-blocks, $\Omega \subset Z^{v}$, we say that a face, $b$, intersects $\Omega$ (written $b \cap \Omega$ ) if at least one of the $L$-blocks separated by $b$ is a subset of $\Omega$. Introduce decoupling parameters $s_{b}$ for all faces $b \cap \Lambda \backslash(\bar{X} \cup \operatorname{supp} C)$ by defining, for each set $\Gamma$ of faces intersecting $\Lambda \backslash(\bar{X} \cup \operatorname{supp} C)$,

$$
s_{x y}(\Gamma)= \begin{cases}s_{b} & \text { if }\{x, y\} \in b \in \Gamma \\ 0 & \text { if }\{x, y\} \in b \notin \Gamma, \quad b \cap \Lambda \backslash(\bar{X} \cup \operatorname{supp} C) . \\ 1 & \text { otherwise }\end{cases}
$$

Denote the Gaussian partition functions obtained from $Z_{q}^{(0)}(\Lambda)$ and $Z_{q}^{(0)}\left(\Lambda_{1} \mid \phi_{\partial X \cup \partial_{e} C}\right)$ upon substituting $s_{x y}(\Gamma)\left(\phi_{x}-\phi_{y}\right)^{2}$ for $\left(\phi_{x}-\phi_{y}\right)^{2}$ by $Z_{q}^{(0)}\left(\Lambda, s_{\Gamma}\right)$ and $Z_{q}^{(0)}\left(\Lambda_{1}, s_{\Gamma} \mid \phi_{\partial X \cup \partial_{e} C}\right)$. Then by the fundamental theorem of calculus,

$$
\frac{Z_{q}^{(0)}\left(\Lambda_{1} \mid \phi_{\partial X \cup \partial_{e} C}\right)}{Z_{q}^{(0)}(\Lambda)}=\frac{Z_{q}^{(0)}\left(\bar{X} \backslash\left(X \cup \partial_{e} C\right) \mid \phi_{\partial X \cup \partial_{e} C}\right)}{Z_{q}^{(0)}(\bar{X} \cup \operatorname{supp} C)} \exp \left(\sum_{\Gamma \neq \phi} W\left(\Gamma \mid \phi_{\partial X \cup \partial_{e} C}\right)\right),
$$

where

$$
W\left(\Gamma \mid \phi_{\partial X \cup \partial_{e} C}\right)=\int_{[0,1]|\Gamma|} d s_{\Gamma} \partial^{\Gamma}\left\{\log Z_{q}^{(0)}\left(\Lambda_{1}, s_{\Gamma} \mid \phi_{\partial X \cup \partial_{e} C}\right)-\log Z_{q}^{(0)}\left(\Lambda, s_{\Gamma}\right)\right\},
$$

with $d s_{\Gamma}=\prod_{b \in \Gamma} d s_{b}, \partial^{\Gamma}=\prod_{b \in \Gamma} \partial / \partial s_{b} ;$ the sum over $\Gamma$ is over all non-empty $\Gamma$ 
intersecting $\Lambda \backslash(\bar{X} \cup \operatorname{supp} C)$. We note, see also [18], that $W(\Gamma \mid \cdot)$ is zero unless $\Gamma$ satisfies the connectivity conditions:

i) $X(\Gamma) \cap(\bar{X} \cup \operatorname{supp} C) \neq \varnothing$,

ii) $X(\Gamma)$ is contained in one and only one connected component of $X(\Gamma) \cup$ $(\bar{X} \cup \operatorname{supp} C)$.

Here, and in the following, $X(\Gamma)$ denotes the union of all $L$-blocks which intersect a face in $\Gamma$. If i) is violated, then the terms from the two partition functions in $(4.8 \mathrm{~b})$ are equal, and thus cancel each other. If ii) is violated, then the derivative $\partial^{\Gamma}$ vanishes because it acts on a sum of terms, one for each connected component of $X(\Gamma) \cup \bar{X} \cup \operatorname{supp} C$, none of which depend on all of the $s_{b}$. Expanding the exponential in (4.8) and inserting the result into Eq. (4.6) we obtain

$$
\begin{aligned}
Z_{q}^{\prime}(\Lambda)= & e^{-e_{q}|\Lambda|} Z_{q}^{(0)}(\Lambda) \sum_{C} \sum_{X \in \Lambda \backslash C} \sum_{T} \frac{1}{\mid \Pi[\mid !} \\
& \cdot \frac{1}{Z_{q}^{(0)}(\bar{X} \cup \operatorname{supp} C)} \int d \phi_{\bar{X}} \exp \left(-H_{q}^{(0)}\left(\phi_{\bar{X}}\right)\right) F_{X}\left(\phi_{X}\right) \\
& \cdot \prod_{\gamma \in C} \exp \left(-\psi_{q}^{\prime}\left(\gamma \mid R_{q}+\phi_{\partial_{e} \gamma}\right)+e_{q}|\operatorname{supp} \gamma|\right) \prod_{\Gamma \in \mathbb{T}} W\left(\Gamma \mid \phi_{\partial X \cup \partial_{e} C}\right),
\end{aligned}
$$

where the last sum is over all sequences $\Pi$ of sets $\Gamma$ satisfying the connectivity conditions i) and ii).

Equation (4.10) is essentially the expansion we are looking for. Defining a polymer in $\Lambda$ as a connected union of $L$-blocks, $P \subset \Lambda$, and its activity

$$
\begin{aligned}
\rho(P)= & \sum_{\substack{C, X, T \text { s.t. } \\
\operatorname{supp} C \cup \bar{X} \cup X(I)=P}} \frac{1}{\mid \bar{\Gamma} !} \frac{1}{Z_{q}^{(0)}(\bar{X} \cup \operatorname{supp} C)} \int d \phi_{\bar{X}} \exp \left(-H_{q}^{(0)}\left(\phi_{\bar{X}}\right)\right) \\
& \cdot \prod_{\gamma \in C} \exp \left(-\psi_{q}^{\prime}\left(\gamma, R_{q}+\phi_{\partial_{e} \gamma}\right)\right) F_{X}\left(\phi_{X}\right) \prod_{\Gamma \in \mathbb{T}} W\left(\Gamma \mid \phi_{\partial X \cup \partial_{e} \Gamma}\right),
\end{aligned}
$$

where $X(\Pi)=\bigcup_{\Gamma \in \mathbb{T}} X(\Gamma)$, we obtain, after rearranging with the help of the multi-nomial theorem,

$$
Z_{q}^{\prime}(\Lambda)=e^{-e_{q}|\Lambda|} Z_{q}^{(0)}(\Lambda) \sum_{\mathbb{P}} \sum_{P \in \mathbb{P}} \rho(P)
$$

where the sum is over sets of unions of $L$-blocks, $\mathbb{P}=\left\{P_{1}, P_{2} \ldots\right\}$, such that each $P_{j}$ is connected and if $j \neq k$ then $P_{j}$ and $P_{k}$ have no face in common.

In the next section we prove the following theorem, from which the convergence of the Mayer expansion for $\log Z_{q}^{\prime}(\Lambda)$ immediately follows.

Theorem 4.1. For all $b<\infty$ one can choose $L_{0}<\infty$ independent of $\beta$ and $L$, and $\gamma_{0}=\gamma_{0}(L), \beta_{1}=\beta_{1}(L), \tau_{1}=\tau_{1}(L)$ not depending on $\beta$, such that

$$
|\rho(P)| \leqq e^{-b\left|P^{\prime}\right|}=e^{-b L^{-v}|P|}
$$

for all polymers $P$, provided $L \geqq L_{0}, \mu \in \mathscr{V}^{(1)}, \tau=\tau(\beta, L) \geqq \tau_{1}$ and $\beta \geqq \beta_{1}$.

Lemma 3.2 is an immediate consequence of Theorem 4.1: Let $\theta_{q}(\Lambda)$ be the 
polymer partition function

$$
\theta_{q}(\Lambda)=\sum_{\mathbb{P}} \prod_{P \in \mathbb{P}} \rho(P)
$$

Choose $b$ large enough, for example so large that the sum $\sum^{\prime}$ over all polymers touching a given $L$-cube $\square\left(x^{\prime}\right)$

$$
\sum_{p}^{\prime} e^{-(b-1)\left|P^{\prime}\right|}<1 .
$$

Then [19] the Mayer expansion for $\log \theta_{q}(\Lambda)$ is absolutely convergent. It follows that the limit

$$
s_{q}=-\lim _{\Lambda \uparrow Z^{v}} \frac{1}{|\Lambda|} \log \theta_{q}(\Lambda)
$$

exists, and that

$$
\left|s_{q}\right| \leqq \varepsilon(b)
$$

and

$$
\left|\log \theta_{q}(\Lambda)+s_{q}\right| \Lambda|| \leqq \varepsilon(b)\left|\partial \Lambda^{c}\right|
$$

for a constant $\varepsilon(b) \rightarrow 0$ as $b \rightarrow \infty$. This, together with the corresponding bounds on the Gaussian partition function $Z_{q}^{(0)}(\Lambda)$-proven for example by random walk methods-immediately gives Lemma 3.2.

\section{Convergence of the Mayer Series for the Truncated Models}

In this section we prove Theorem 4.1. The proof will follow from two lemmas. We first state the lemmas. Then using them we prove Theorem 4.1. Finally, we prove the two lemmas themselves.

Lemma 5.1. $\forall c<\infty$ there is a constant $L_{0}$ (depending on $c, \eta, \eta^{\prime \prime}$ and $v$ ) such that, $\forall L \geqq L_{0}, \forall \varepsilon>0$ and a suitable choice of $r_{0}$ (depending on $\varepsilon, L, \eta, \eta^{\prime \prime}$ and $v$ ),

$$
\begin{aligned}
& \sum_{\substack{\mathbb{T} \text { s.t. } \\
\text { supp } C \cup \bar{X} \cup X(T)=P}} \frac{1}{|\bar{\Gamma}| !} \prod_{\Gamma \in \mathbb{T}}\left|W\left(\Gamma \mid \phi_{\partial X \cup \partial_{e} c}\right)\right| \\
& \leqq \exp \left\{-c L^{-v}|P \backslash(\bar{X} \cup \operatorname{supp} C)|+f_{1}|\bar{X} \cup \operatorname{supp} C|+\frac{\varepsilon}{2} \sum_{x \in \partial X \cup \partial_{e} C} \phi_{x}^{2}\right\},
\end{aligned}
$$

$f_{1}$ is a constant depending only on $L, v, \eta$ and $\eta^{\prime \prime}$.

Lemma 5.2. $\forall \tilde{c}<\infty, \forall \varepsilon<\eta$ there is a choice of $\beta_{1}$, (depending on $\tilde{c}, \varepsilon, v, \eta, \eta^{\prime}, \eta^{\prime \prime}$ ) such that

$$
\begin{aligned}
& \frac{1}{Z_{q}^{(0)}(\bar{X} \cup \operatorname{supp} C)} \int\left|F_{X}\left(\phi_{X}\right)\right| \exp \left(-H_{q}^{(0)}\left(\phi_{\bar{X}}\right)+\frac{\varepsilon}{2} \sum_{x \in \partial X \cup \partial_{e} C} \phi_{x}^{2}\right) d \phi_{\bar{X}} \\
& \quad \leqq \exp \left\{-\tilde{c}|x|+f_{2}\left|X \cup \operatorname{supp} C \cup \partial_{e} C\right|,\right.
\end{aligned}
$$

provided $\beta \geqq \beta_{1}$. The constant $f_{2}$ depends only on $\varepsilon, v, \eta, \eta^{\prime}$ and $\eta^{\prime \prime}$. 
5.1 Proof of Theorem 4.1. We choose $\varepsilon=\eta / 2, c=b+\log 2$, and $\tau_{1}=3 \tilde{c}_{1}$, and we fix $L \geqq L_{0}$ and $r_{0}$ according to Lemma 5.1. $\tilde{c}>b$ will be chosen later. Using the bound

$$
\exp \left(-\psi_{q}^{\prime}\left(\gamma \mid R_{\partial_{e} C}\right)+e_{q}|\operatorname{supp} \gamma|\right) \leqq \exp \left(-\frac{\tau}{3}|\operatorname{supp} C|\right)
$$

together with Lemma 5.1, Lemma 5.2 and the fact that $\left|\partial_{e} C\right| \leqq\left(3^{v}-1\right)|\operatorname{supp} C|$ we obtain

$$
\begin{aligned}
|\rho(P)| \leqq & \sum_{\substack{C, X \text { s.t. } \\
\bar{X} \cup \operatorname{supp} C \subset P}} \exp \left(-c L^{-v}|P|+\left(f_{1}+c L^{-v}\right)|\bar{X} \cup \operatorname{supp} C|\right) \\
& \cdot \exp \left(-\left(c-3^{v} f_{2}\right)|X \cup \operatorname{supp} C|\right),
\end{aligned}
$$

provided $\tau \geqq \tau_{1}=3 \tilde{c}$ and $\beta \geqq \beta_{1}=\beta_{1}(\tilde{c})$. Next we use the definition of $\bar{X}$ to conclude that

$$
|\bar{X}| \leqq\left(2 r_{0}+1\right)^{v}\left|X \cup \partial_{e} C\right| \leqq\left(2 r_{0}+1\right)^{v}\left(|X|+\left(3^{v}-1\right)|\operatorname{supp} C|\right),
$$

which shows that

$$
|X \cup \operatorname{supp} C| \geqq 3^{-v}\left(2 r_{0}+1\right)|\bar{X} \cup \operatorname{supp} C| .
$$

Bounding the sum over $C$ and $X$ with fixed "support," $Y=\bar{X} \cup \operatorname{supp} C$, by $2^{|Y|}(N+1)^{|Y|}$, choosing

$$
\tilde{c}=3^{v} f_{2}+\left[f_{1}+c L^{-v}+\log (2 N+2)\right]\left(6 r_{0}+3\right)^{v}
$$

and bounding the remaining sum by $2^{|P| L^{-v}}$ we obtain Theorem 4.1 .

5.2 Proof of Lemma 5.1. Using the fact that the contributions from $\Lambda \backslash P$ cancel in the definition of $W\left(\Gamma \mid \phi_{\partial X \cup \partial_{e} C}\right)$ we rewrite

$$
W\left(\Gamma \mid \phi_{\partial X \cup \partial_{e} C}\right)=\int d s_{\Gamma} \partial^{\Gamma}\left\{\log Z_{q}^{(0)}\left(P_{1}, s_{\Gamma} \mid \phi_{\partial X \cup \partial_{e} C}\right)-\log Z_{q}^{(0)}\left(P, s_{\Gamma}\right)\right\}
$$

with $P_{1}=P \backslash\left(X \cup \operatorname{supp} C \cup \partial_{e} C\right)$. Let $C^{(2)}\left(s_{\Gamma}\right)$ be the covariance matrix corresponding to $Z_{q}^{(0)}\left(P, s_{\Gamma}\right)$, i.e.

$$
C^{(2)}\left(s_{\Gamma}\right)=\left\langle\phi_{x} \phi_{y}\right\rangle_{P, s_{\Gamma}}^{0}-\left\langle\phi_{x}\right\rangle_{P, s_{\Gamma}}^{(0)}\left\langle\phi_{y}\right\rangle_{P, s_{\Gamma}}^{(0)},
$$

where \langle\rangle$_{P, s_{\Gamma}}^{(0)}$ denotes the expectation with respect to the Gibbs factor $\exp \left(-H_{q}^{(0)}\left(\phi_{P}, s_{\Gamma}\right)\right)$ obtained from $c^{-H_{q}^{(0)}\left(\phi_{P}\right)}$ by substituting $s_{x y}(\Gamma)\left(\phi_{x}-\phi_{y}\right)^{2}$ for $\left(\phi_{x}-\phi_{y}\right)^{2}$, and let $C^{(1)}\left(s_{\Gamma}\right)$ be the covariance matrix corresponding similarly to $Z_{q}^{(0)}\left(P_{1}, s_{\Gamma} \mid \phi_{\partial X \cup \partial_{e} C}\right)$. Evaluating the Gaussian partition function in (5.1) we rewrite $W(\Gamma \mid \cdot)$ as

with

$$
W\left(\Gamma \mid \phi_{\partial X \cup \partial_{e} C}\right)=W(\Gamma)+\frac{1}{2} \sum_{x, y \in \partial X \cup \partial_{e} C} \phi_{x} B_{x y}(\Gamma) \phi_{y}
$$

$$
\begin{aligned}
W(\Gamma) & =\frac{1}{2} \int d s_{\Gamma} \partial^{\Gamma}\left\{\operatorname{tr} \log C^{(1)}\left(s_{\Gamma}\right)-\operatorname{tr} \log C^{(2)}\left(s_{\Gamma}\right)\right\} \\
B_{x y}(\Gamma) & =\sum_{\substack{u, v \in P \\
|x-v|=1 \\
y-u \mid=1}} \int d s_{\Gamma} \partial^{\Gamma} C_{u v}^{(1)}\left(s_{\Gamma}\right)
\end{aligned}
$$


Our starting point is the following basic estimate (proven e.g. in [18])

$$
\left|\partial^{\Gamma} C_{x y}^{(1)}\right| \leqq d_{0}^{|\Gamma|+1} \exp \left(-m_{0} d(x, \Gamma, y)\right),
$$

where $d_{0}<\infty, m_{0}>0$ are constants depending only on $\eta, \eta^{\prime \prime}$ and $v$ (recall that $\left.m_{q}^{2} \geqq \eta, \kappa_{q} \leqq \eta^{\prime \prime}\right)$ and $d(x, \Gamma, y)$ is the length of the shortest path from $x$ to $y$ that visits each bond $b \in \Gamma$ at least once.

Proposition 5.3. Given $\eta, \eta^{\prime \prime}$ and $v$ there is a constant $d_{1}<\infty$ such that

$$
\sum_{\Gamma \text { s.t. }\{\Gamma \mid=n}\left|W\left(\Gamma, \phi_{\partial X \cup \partial_{e} C}\right)\right| \leqq\left(d_{1} e^{-\left(m_{0} L / 4 v\right)}\right)^{n}\left[f_{1}|\bar{X} \cup \operatorname{supp} C|+\frac{\varepsilon_{1}}{2} \sum_{x \in \partial X \cup \partial_{e} C} \phi_{x}^{2}\right] \text {, }
$$

where

$$
f_{1}=\left(d_{1} / L\right) e^{L m_{0} / 2}, \quad \varepsilon_{1}=d_{1} e^{-m_{0}\left(r_{0}-L / 2\right)} .
$$

Proof. We first bound $|W(\Gamma)|$. Fixing a bond $b \in \Gamma$ and using the formula

$$
\frac{\partial}{\partial s_{b}} C^{(i)}=-C^{(i)} \frac{\partial\left[C^{(i)}\right]^{-1}}{\partial s_{b}} C^{(i)}
$$

we obtain, noting that

$$
I_{b}=-\frac{\partial}{\partial s_{b}}\left(C^{(1)}\right)^{-1}=-\frac{\partial}{\partial s_{b}}\left(C^{(2)}\right)^{-1}
$$

does not depend on $s_{\Gamma}$,

$$
W(\Gamma)=\frac{1}{2} \int d s_{\Gamma}\left[\operatorname{tr} I_{b} \partial^{\Gamma \backslash\{b\}} C^{(2)}-\operatorname{tr} I_{b} \partial^{\Gamma \backslash\{b\}} C^{(1)}\right] .
$$

Using (5.4) together with the explicit representation

$$
\left(I_{b}\right)_{x y}=\kappa_{q} \sum_{\{u, v\}\} b}\left\{\delta_{u x} \delta_{v y}+\delta_{v x} \delta_{u y}-\delta_{u x} \delta_{u y}-\delta_{v x} \delta_{v y}\right\}
$$

and the bound $\kappa_{q} \leqq \eta^{\prime \prime}$ we obtain

$$
|W(\Gamma)| \leqq 4 \eta^{\prime \prime} L^{v-1} d_{0}|\Gamma| e^{-m_{0} d(\Gamma)},
$$

where $d(\Gamma)$ is the length of the shortest closed path that visits each face in $\Gamma$ at least once. Using the fact that $W(\Gamma)=0$ if $X(\Gamma) \cap(\bar{X} \cup \operatorname{supp} C)=\varnothing$ we can always label the bonds $b_{1}, \ldots, b_{n} \in \Gamma$ in such a way that

i) $b_{1} \cap \square\left(x^{\prime}\right) \neq \varnothing$ for some $\square\left(x^{\prime}\right) \subset(\bar{X} \cup \operatorname{supp} C)$,

ii) $d(\Gamma) \geqq \sum_{i=1}^{n-1} \operatorname{dist}\left(b_{i}, b_{i+1}\right)$.

Bounding the sum over sets $\Gamma$ by a sum over sequences $b_{1}, \ldots, b_{n}$ we get

$$
\begin{aligned}
\sum_{\substack{\Gamma \text { s.t. } \\
|\Gamma|=n}}|W(\Gamma)| \leqq & L^{-v}|\bar{X} \cup \operatorname{supp} C| 4 \eta^{\prime \prime} L^{\nu-1} d_{0}^{n} \cdot e^{L m_{0} / 2} e^{-n\left(L m_{0} / 4 v\right)} \\
& \cdot \sup _{x^{\prime}} \sum_{\substack{b_{1}, ., b_{\eta} \text { s.t. } \\
b_{1} \cap \square(x) \neq \phi}} \exp \left(-\frac{m_{0}}{2} \sum_{i=1}^{n-1} \operatorname{dist}\left(b_{i}, b_{i+1}\right)\right),
\end{aligned}
$$

where we have used the bound $d(\Gamma) \geqq(|\Gamma| / 2 v-1) L$ (for a proof, see e.g. 
[18]) to extract the factor $e^{L m_{0} / 2} e^{-|\Gamma| L m_{0} / 4 v}$. We now use the fact that there are not more than $2 v$ bonds, $b_{1}$, with $\square\left(x^{\prime}\right) \cap b_{1} \neq \varnothing$, together with the bound $\sum_{b} \exp \left(\left(-m_{0} / 2\right) \operatorname{dist}\left(b, b^{\prime}\right)\right) \leqq K$, where $K<\infty$ is a constant depending only on $v$ and $m_{0}$, to get the estimate

$$
\sum_{\substack{\Gamma \text { s.t. } \\|\Gamma|=n}}|W(\Gamma)| \leqq|\operatorname{supp} C \cup \bar{X}|\left(8 v \eta^{\prime \prime} / L\right) e^{L m_{0} / 2}\left(K d_{0} e^{-L m_{0} / 4 v}\right)^{n} .
$$

On the other hand, by (5.4) and the definition of $B_{x y}(\Gamma)$,

$$
\begin{aligned}
\sum_{|\Gamma|=n} \sum_{y \in \partial X \cup \partial_{e} C}\left|B_{x y}(\Gamma)\right| \leqq & 4 v^{2} d_{0}^{n+1} e^{L m_{0} / 2} e^{-\left(L m_{0} / 4 v\right) n} \\
& \cdot e^{-m_{0} r_{0}} \sup _{u} \sum_{|\Gamma|=n} \sum_{v} \exp \left(\frac{-m_{0}}{2} d(u, \Gamma, v)\right),
\end{aligned}
$$

where we have used the fact that

$$
\operatorname{dist}(x, \Gamma) \geqq r_{0}, \quad \operatorname{dist}(y, \Gamma) \geqq r_{0}
$$

to extract the additional factor $e^{-m_{0} r_{0}}$. Bounding the sum over $\Gamma$ with $|\Gamma|=n$ as before, and noticing that

$$
\left|\phi_{x} \phi_{y}\right| \leqq \frac{1}{2}\left(\phi_{x}^{2}+\phi_{y}^{2}\right)
$$

which implies that

we find

$$
\sum_{x, y \in \partial X \cup \partial_{e} C}\left|\phi_{x} B_{x y} \phi_{y}\right| \leqq \sum_{x \in \partial X \cup \partial_{e} C} \phi_{x}^{2} \sum_{y \in \partial X \cup \partial_{e} C}\left|B_{x y}\right|,
$$

$$
\sum_{x, y \in \partial X \cup \partial_{e} C} \sum_{\substack{\Gamma \text { s.t. } \\|\Gamma|=n}}\left|\phi_{x} B_{x y}(\Gamma) \phi_{y}\right| \leqq\left(k d_{0} e^{-L m_{0} / 4 v}\right)^{n} \cdot 4 v^{2} d_{0} e^{L m_{0} / 2-m_{0} r} K \sum_{x \in \partial X \cup \partial_{e} C} \phi_{x}^{2} .
$$

Choosing

$$
d_{1}=\max \left\{K d_{0}, 8 v \eta^{\prime \prime}, 8 v^{2} K d_{0}\right\},
$$

we obtain the proposition.

We now prove Lemma 5.1. Given $c$ we choose $L_{0}$ so large that

$$
d_{1} e^{-m_{0} L_{0} / 4 v} \leqq \frac{1}{2} e^{-2 c} .
$$

Using the fact that $X(\Gamma) \cup \bar{X} \cup \operatorname{supp} C=P$, together with Proposition 5.3 and the bound $|\Gamma| \geqq \frac{1}{2} L^{-v}|X(\Gamma)|$ we may extract a factor

$$
\prod_{\Gamma \in \mathbb{T}} e^{-2 c|\Gamma|} \leqq \exp \left(-c L^{-v}|P \backslash(\bar{X} \cup \operatorname{supp} C)|\right)
$$

from the left-hand side of Lemma 5.1 and bound the remaining sum by

$$
\begin{aligned}
& \sum_{k=0}^{\infty} \frac{1}{k !}\left\{\left[f_{1} L^{-v}|\bar{X} \cup \operatorname{supp} C|+\frac{\varepsilon_{1}}{2} \sum_{x \in \partial X \cup \partial_{e} C} \phi_{x}^{2}\right] \sum_{n=1}^{\infty}\left(\frac{1}{2}\right)^{n}\right\}^{k} \\
& \quad=\exp \left[f_{1} L^{-v}|\bar{X} \cup \operatorname{supp} C|+\frac{\varepsilon_{1}}{2} \sum_{x \in \partial X \cup \partial_{e} C} \phi_{x}^{2}\right],
\end{aligned}
$$

which proves Lemma 5.1 (choose $r_{0}$ so large that $\varepsilon_{1}=\varepsilon_{1}\left(r_{0}, L\right) \leqq \varepsilon$ ). 
5.3 Proof of Lemma 5.2. We first bound, using the fact that $W \geqq 0$,

$$
\begin{aligned}
& \frac{1}{Z_{q}^{(0)}(\bar{X} \cup \operatorname{supp} C)} \int d \phi_{\bar{X}} F_{X}\left(\phi_{X}\right) \exp \left(-H_{q}^{(0)}\left(\phi_{\bar{X}}\right)+\frac{\varepsilon}{2} \sum_{x \in \partial X \cup \partial_{e} C} \phi_{x}^{2}\right) \\
& \leqq \frac{Z_{q}^{(0)}\left(\bar{X} \backslash\left(X \cup \partial_{e} C\right)\right)}{Z_{q}^{(0)}(\bar{X} \cup \operatorname{supp} C)} \int d \phi_{X \cup \partial_{e} C} F_{X}\left(\phi_{X}\right) \exp \left(-H_{q}^{(0)}\left(\phi_{X \cup \partial_{e} C}\right)+\frac{\varepsilon}{2} \sum_{x \in \partial X \cup \partial_{e} C} \phi_{x}^{2}\right) \\
& \leqq \exp \left(\tilde{f}_{2}\left|X \cup \operatorname{supp} C \cup \partial_{e} C\right|\right) \int d \phi_{X \cup \partial_{e} C} F_{X}\left(\phi_{X}\right) \\
& \cdot \exp \left(-H_{q}^{(0)}\left(\phi_{X \cup \partial_{e} C}\right)+\frac{\varepsilon}{2} \sum_{x \in \partial X \cup \partial_{e} C} \phi_{x}^{2}\right),
\end{aligned}
$$

where $\tilde{f}_{2}$ is a constant depending only on $v, \eta, \eta^{\prime}$ and $\eta^{\prime \prime}$. Using the fact that the sum in the definition (4.3c) of $F_{X}\left(\phi_{X}\right)$ contains at most $2^{|X|} 2^{2 v|X|}$ terms, we are left with the proof of the following proposition (choose $Y=X \cup \partial_{e} C$ and $f_{2}=\tilde{f}_{2}+$ $\left.(2 v+1) \log 2+\frac{1}{2}|\log (2 \pi / \tilde{\eta})|\right)$.

Proposition 5.4. $\forall \tilde{c}<\infty \forall \varepsilon<\eta$ there is a choice of $\beta_{1}$ (depending on $\tilde{c}, \varepsilon, \eta, \eta^{\prime}$ and $\left.\eta^{\prime \prime}\right)$ such that

$$
\begin{aligned}
& \int d \phi_{y}\left|\prod_{x \in X_{1}} F_{x}\left(\phi_{x}\right) \prod_{\langle x y\rangle \in B} F_{x y}\left(\phi_{x}, \phi_{y}\right)\right| \exp \left(-H_{q}^{(0)}\left(\phi_{Y}\right)+\frac{\varepsilon}{2} \sum_{x \in Y} \phi_{x}^{2}\right) \\
& \leqq\left(\frac{2 \pi}{\tilde{\eta}}\right)^{(1 / 2)|Y|} \exp \left(-\tilde{c}\left|X_{1} \cup X(B)\right|\right),
\end{aligned}
$$

provided $X_{1} \cup X(B) \subset Y . \tilde{\eta}$ is the constant $\eta-\varepsilon$.

Proof. We introduce the notation

$$
\begin{aligned}
G_{x y} & =\left|F_{x y}\left(\phi_{x}, \phi_{y}\right)\right| \exp \left(-\frac{\kappa_{q}}{2}\left|\phi_{x}-\phi_{y}\right|^{2}\right), \\
G_{x} & =\exp \left(-\frac{m_{q}^{2}-\varepsilon}{2} \phi_{x}^{2}\right)\left\{\begin{array}{lll}
1 & \text { if } & x \notin X_{1} \\
\left|F_{x}\left(\phi_{x}\right)\right| & \text { if } & x \notin X_{1}
\end{array}\right.
\end{aligned}
$$

and $\tilde{\eta}=\eta-\varepsilon$. The left-hand side of (5.8) can be bounded by

$$
\begin{aligned}
& \int \prod_{\langle x y\rangle \in B} G_{x y}\left(\phi_{x}, \phi_{y}\right) \prod_{x \in Y} G_{x}\left(\phi_{x}\right) d \phi_{x} \\
& \leqq \prod_{x \in Y}\left[\int d \phi_{x} G_{x}\right]^{1-n_{x} / 2 v} \prod_{\langle x y\rangle \in B}\left[\int d \phi_{x} d \phi_{y} G_{x} G_{x y}^{2} G_{y}\right]^{1 / 2 v},
\end{aligned}
$$

where we used Hölder's inequality and the fact that each point in $Z^{v}$ can be shared by at most $2 v$ nearest neighbor pairs. $n_{x}$ is the number of nearest neighbor pairs in $B$ that contain the point $x$. The integrals in (5.9) are estimated using the following bounds (they all follow from assumption A.1 and the definition of $G_{x}, G_{x y}$ ):

if $x \in X_{1}$ and $\left|\phi_{x}\right| \leqq \xi(\beta)$,

$$
\left|G_{x}\left(\phi_{x}\right)\right| \leqq\left(e^{\delta(\beta)}-1\right) e^{-(\tilde{\eta} / 2) \phi_{x}^{2}}
$$

$$
\left|G_{x}\left(\phi_{x}\right)\right| \leqq e^{(\varepsilon / 2) \phi_{x}^{2}}\left[\exp \left(-\left(V\left(\phi_{x}+R_{q}\right)-e_{q}\right)\right)+\exp \left(-\frac{m_{q}^{2}}{2} \phi_{x}^{2}\right)\right] \leqq 2 e^{-(\hat{\eta} / 2) \phi_{x}^{2}}
$$


if $x \in X_{1}$ and $\left|\phi_{x}\right| \geqq \xi(\beta)$,

if $x \in Y \backslash X_{1}$,

$$
\left|G_{x}\left(\phi_{x}\right)\right| \leqq 2 e^{-(\tilde{\eta} / 2) \phi_{x}^{2}}
$$

$$
\left|G_{x y}\left(\phi_{x} \phi_{y}\right)\right| \leqq e^{\delta(\beta)}-1
$$

if $\left|\phi_{x}\right| \leqq \xi(\beta)$ and $\left|\phi_{y}\right| \leqq \xi(\beta)$, and

$$
\left|G_{x y}\left(\phi_{x}, \phi_{y}\right)\right| \leqq 1,
$$

if $\left|\phi_{x}\right|>\xi(\beta)$ or $\left|\phi_{y}\right|>\xi(\beta)$.

Combined with (5.9) and the estimates

$$
\begin{aligned}
& \int_{-\xi(\beta)}^{\xi(\beta)} d \phi_{x} e^{-(\tilde{\eta} / 2) \phi_{x}^{2}} \leqq \int_{-\infty}^{\infty} d \phi_{x} e^{-(\tilde{\eta} / 2) \phi_{x}^{2}}=\frac{\sqrt{2 \pi}}{\tilde{\eta}} \\
& \int_{\left|\phi_{x}\right| \geqq \xi(\beta)} e^{-(\tilde{\eta} / 2) \phi_{x}^{2}} \leqq \frac{\sqrt{2 \pi}}{\tilde{\eta}} e^{-(\tilde{\eta} / 2) \xi(\beta)^{2}},
\end{aligned}
$$

we obtain the bound

$$
\left[2 \frac{\sqrt{2 \pi}}{\tilde{\eta}}\right]^{|Y|}\left[\frac{1}{2}\left(e^{\delta(\beta)}-1\right)+e^{-(\tilde{\eta} / 2) \xi(\beta)^{2}}\right]^{\left|X_{1}\right|} \times\left[\left(e^{\delta(\beta)}-1\right)^{2 v}+2 e^{-(\tilde{\eta} / 2) \xi(\beta)^{2}}\right]^{|B| / 2 v}
$$

for the left-hand side of (5.8). Using the fact that $|X(B)| \leqq 2|B|$ and that $e^{-(\eta / 2) \xi^{2}(\beta)} \leqq \delta(\beta)$, we obtain the proposition with $e^{-c}=O\left(\delta(\beta)^{\lambda}\right)$, for some $\lambda>0$ depending on $(\eta-\varepsilon) / \eta$ and $v$ (for $\varepsilon=\eta / 2, \lambda=1 / 8 v$ ).

\section{The Phase Diagram}

In Sect. 3 it was shown that the free energies of the truncated contour models,

$$
h_{q}^{\prime}=\lim _{\Lambda \uparrow Z^{v}} \frac{1}{|\Lambda|} \log Z_{q}^{\prime}(\Lambda),
$$

are enough to determine which phases, $q$, are stable in the sense that $\psi_{q}^{\prime}=\psi_{q}$ (which implies, as we show in the next section, that infinite volume Gibbs states, $\langle\cdot\rangle_{q^{\prime}}$ can be constructed for such $q$ ). The stable $q$ 's are those for which $h_{q}^{\prime}$ is minimal. Thus, recalling Eq. (3.8), the $a_{q}$ indicates which phases are stable: $q$ is stable iff $a_{q}=0$. If $q$ is not stable then $a_{q}>0$ so that $a_{q}$ determines a function from $\mathscr{V}^{(1)} \subset R^{N-1}$ into $R_{0}^{N}=\left\{x \in R^{N} \mid \forall k, x_{k} \geqq 0\right.$ and $\exists j$ with $\left.x_{j}=0\right\}$. In the Pirogov-Sinai theory the inverse of this function is called the phase diagram [9]. The standard physicist's notion of the phase diagram corresponds to the images, in $\mathscr{V}^{(1)}$, of the various hyperplanes given by $x_{j_{1}}=x_{j_{2}}=\cdots=x_{j_{m}}=0$ for all possible non-empty $\left\{j_{1}, \ldots, j_{m}\right\} \subset\{1,2, \ldots, N\}$. These consist of all $\mu \in \mathscr{V}^{(1)}$ for which the phases $j_{1}, \ldots, j_{m}$ are stable.

In order to simplify the presentation we prefer to do away with $R_{0}^{N}$ by considering, rather than $a_{q}$, the function

$$
b=\left(\begin{array}{c}
h_{1}-h_{N} \\
h_{2}-h_{N} \\
\vdots \\
h_{N-1}-h_{N}
\end{array}\right)
$$


which maps $\mathscr{V}^{(1)}$ into $R^{N-1}$. (Note that there is nothing special about $N$ in Eq. (6.1). We could have chosen any $j \in\{1, \ldots, N\}$ and defined $b$ by $b_{k}=h_{k}-h_{j}$.) The phase diagram is here given by the images, under $b^{-1}$, of the sets $\left\{y \in R^{N-1} \mid y_{j} \geqq 0\right.$ for all $\left.j, y_{j_{1}}, \ldots, y_{j_{m}}=0\right\}$ for all possible $\left\{j_{1}, \ldots, j_{m}\right\} \subset\{1, \ldots, N-1\}$ (including the empty set as a possibility) and the sets $\left\{y \in R^{N-1} \mid y_{j_{1}}=y_{j_{2}}=\cdots=y_{j_{m}}<0\right\}$ for all possible non-empty $\left\{j_{1}, \ldots, j_{m}\right\} \subset\{1, \ldots, N-1\}$. The advantage of using $b$ is that it simplifies the following

Theorem 6.1. If $\beta$ is large enough then there exist open sets, $\mathscr{V}, \mathscr{W} \subset R^{N-1}$ such that

i) $b: \mathscr{V} \rightarrow \mathscr{W}$ is a homeomorphism,

ii) $O \in \mathscr{W}$.

Comments. The point of (i) is that $b$ is in fact continuously invertible. (ii) shows that the point of maximal phase co-existence, when all phases, $q$, are stable, is in the phase diagram. This insures that all possible combinations of co-existing phases can be obtained by varying $\mu$ in $\mathscr{V}$. It is also possible to demonstrate that, for $\beta$ large enough, $0 \in \mathscr{V} . b(0)$ is not, however, in general 0.

Let $f_{j}(\mu)=e_{j}(\mu)-e_{N}(\mu)$ for $j \in\{1,2, \ldots, N-1\}$ and let $s_{j}(\mu)=h_{j}(\mu)-h_{N}(\mu)-f_{j}(\mu)$. Then Eq. (6.1) reads

$$
b=f(\mu)+s(\mu) .
$$

By Lemma $3.2\left|s_{j}(\mu)\right| \leqq 2 c_{1}$ for all $\mu \in \mathscr{V}^{(1)}$, where $\mathscr{V}^{(1)}$ is the set defined in Lemma 3.1. By assumption (A.4) and the inverse function theorem there is an open set $\mathscr{W}^{(1)} \subset R^{N-1}$ containing a sphere of radius $M(\beta)^{1-\lambda}$ around 0 such that $\mathscr{V}^{(1)}$ and $\mathscr{W}^{(1)}$ are diffeomorphic under the action of $f$. Let

$$
\mathscr{W}=\left\{b \in \mathscr{W}^{(1)} \mid b^{\prime} \in \mathscr{W}^{(1)} \forall b^{\prime} \text { s.t. }\left|b^{\prime}-b\right| \leqq 2 N c_{1}\right\} .
$$

Then, $\forall b \in \mathscr{W}$ and all $k \geqq 1, \mu^{(0)}=f^{-1}(b)$ and $\mu^{(k)}=f^{-1}\left(b-s\left(\mu^{(k-1)}\right)\right)$ are well defined, with $\mu^{(k)} \in \mathscr{V}^{(1)}$.

Again, assumption A.4 and Lemma 3.2 allow us to conclude that, for $b \in \mathscr{W}$ and $\mu, \mu^{\prime} \in \mathscr{V}^{(1)}$,

$$
\begin{array}{r}
\left|\mu^{(0)}\right|=\left|f^{-1}(b)\right| \leqq \frac{1}{M(\beta)}|b| \\
\left|\mu^{(0)}-\mu^{(1)}\right| \leqq \frac{1}{M(\beta)}\left|s\left(\mu^{(0)}\right)\right| \leqq \frac{2 N c_{1}}{M(\beta)}
\end{array}
$$

and

$$
\left|f^{-1}(b-s(\mu))-f^{-1}\left(b-s\left(\mu^{\prime}\right)\right)\right| \leqq \frac{1}{M(\beta)}\left|s(\mu)-s\left(\mu^{\prime}\right)\right| .
$$

Therefore, the existence of the limit $\mu=\lim _{k \rightarrow \infty} \mu^{(k)}$ solving Eq. (6.2), as well as the continuity of the resulting $b^{-1}$ follows from the following estimate.

Lemma 6.2. There is a constant $c(\beta)$ such that

$$
\lim _{\beta \rightarrow \infty} \frac{c(\beta)}{M(\beta)}=0
$$


and, for all $\mu, \mu^{\prime} \in \mathscr{V}^{(1)}$

$$
\left|s(\mu)-s\left(\mu^{\prime}\right)\right| \leqq c(\beta)\left|\mu-\mu^{\prime}\right| .
$$

Proof. Letting $h_{q, \Lambda}^{\prime}(\mu)=(1 /|\Lambda|) \log Z_{q}^{\prime}(\Lambda)$ it is enough to bound the one sided derivatives $\left(d / d \mu_{ \pm}\right)\left(h_{q, \Lambda}^{\prime}(\mu)-e_{q}(\mu)\right)$ uniformly in $\Lambda$ for all $q$. Note that

$$
\frac{d}{d \mu_{ \pm}} h_{q, \Lambda}^{\prime}(\mu)=\frac{1}{|\Lambda|}\left(g_{q}^{(1)}(\mu)+g_{q}^{(2)}(\mu)\right)
$$

where

$$
g_{q}^{(1)}(\mu)=\frac{-1}{Z_{q}^{\prime}(\Lambda)} \sum_{C} \int d R_{\Lambda \backslash C} \exp \left(-\psi_{q}^{\prime}\left(C \mid R_{\partial_{e} C}\right)\right) \frac{d}{d \mu_{ \pm}} \exp \left(-H\left(R_{\Lambda \backslash C}\right)\right) \chi_{q}\left(R_{\Lambda \backslash C}\right)
$$

and

$$
g_{q}^{(2)}(\mu)=\frac{-1}{Z_{q}^{\prime}(\Lambda)} \sum_{C} \int d R_{\Lambda \backslash C} \exp \left(-H\left(R_{\Lambda \backslash C}\right)\right) \chi_{q}\left(R_{\Lambda \backslash C}\right) \frac{d}{d \mu_{ \pm}} \exp \left(-\psi_{q}^{\prime}\left(C \mid R_{\partial_{e} C}\right)\right) .
$$

We find

$$
\begin{aligned}
g_{q}^{(1)}(\mu)= & \frac{1}{Z_{q}^{\prime}(\Lambda)} \sum_{x \in \Lambda} \sum_{C \text { s.t. } x \notin \text { supp } C} \int d R_{\Lambda \backslash C} \chi_{q}\left(R_{\Lambda \backslash C}\right) \\
& \cdot \exp \left(-\psi_{q}^{\prime}\left(C \mid R_{\partial_{e} C}\right)-H\left(R_{\Lambda \backslash C}\right)\right) \frac{d}{d \mu}\left(V\left(R_{x}\right)+\frac{1}{2} \sum_{y \in \Lambda \backslash C \text { s.t. }|x-y|=1} W\left(R_{x}, R_{y}\right)\right) .
\end{aligned}
$$

Using assumption A.5 to estimate derivatives, the bound (3.1) (with $\tau / 3$ instead of $\tau$ ) for all contours $\gamma$ with $x \in \operatorname{Int} \gamma$ and $W \geqq 0$ to eliminate couplings between partition functions we can re-sum the remaining contours and obtain

$$
\begin{aligned}
& \left|g_{q}^{(1)}(\mu)-\right| \Lambda\left|\frac{d e_{q}(\mu)}{d \mu}\right| \leqq(2 v+1)(N-1) K_{6}(\beta) \\
& \cdot \sum_{x^{\prime} \in \Lambda^{\prime}} \int d R_{\square\left(x^{\prime}\right)} \chi_{q}\left(R_{\square\left(x^{\prime}\right)}\right)\left(\sum_{x \in \square\left(x^{\prime}\right)}\left|R_{x}-R_{q}\right|^{p}\right) \exp \left(-\sum_{x \in \square\left(x^{\prime}\right)}\left(e_{q}+\frac{\eta}{2}\left(R_{x}-R_{q}\right)^{2}\right)\right) \\
& \cdot \sum_{\substack{C \text { s.t. } \\
\square\left(x^{\prime}\right) \leq \text { Int } \gamma \\
\forall \gamma \in C}} \frac{\left.Z_{q}^{\prime}(\Lambda)\left(\operatorname{supp} C \cup \square\left(x^{\prime}\right)\right)\right)}{Z_{q}^{\prime}(\Lambda)} \prod_{\gamma \in C} \exp \left(-\left(\frac{\tau}{3}+e_{q}\right)|\operatorname{supp} \gamma|\right),
\end{aligned}
$$

where $\eta$ is the constant from assumption A.1. The existence of a convergent cluster expansion for $Z_{q}^{\prime}(\cdot)$ allows us to estimate

$$
\frac{Z_{q}^{\prime}\left(\Lambda \backslash\left(\square\left(x^{\prime}\right) \cup \operatorname{supp} C\right)\right)}{Z_{q}^{\prime}(\Lambda)} \leqq \exp \left(\left(e_{q}+c_{1}\right)\left|\square\left(x^{\prime}\right) \cup \operatorname{supp} C\right|\right),
$$

where $c_{1}$ is the constant from Lemma (3.2). Thus, for $\tau$ large enough, we may bound the sum over $C$ by $1+O\left(e^{-(1 / 3) \tau}\right) \leqq 2$, obtaining $\left|g_{q}^{(1)}(\mu)-\right| \Lambda\left|\frac{d e_{q}(\mu)}{d \mu}\right| \leqq|\Lambda|(2 v+1)(N-1)\left(\frac{2 \pi}{\eta}\right)^{L^{v}-1} K_{6}(\beta) \cdot e^{c_{1} L^{v}} \cdot 2 \int d R|R|^{p} e^{-(1 / 2) \eta R^{2}}$

To estimate $g_{q}^{(2)}(\mu)$ we again use $W \geqq 0$ to decouple, and bound ratios of partition 
functions as in the step preceding Eq. (6.4) to obtain, where $\sum^{\prime}$ is the sum over sets $C$ of pairwise compatible contours, $\gamma^{\prime}$, all compatible with $\gamma$, such that $\operatorname{supp} \gamma \subset \operatorname{int} \gamma^{\prime}$,

$$
\begin{aligned}
\left|g_{q}^{(2)}(\mu)\right| & \leqq \sum_{\gamma} \sum_{C}^{\prime}\left|\frac{d}{d \mu_{ \pm}} \exp \left(-\psi_{q}^{\prime}\left(\gamma \mid R_{\partial_{e}}\right)\right)\right| \cdot \exp \left(\left(e_{q}+c_{1}\right)|\operatorname{supp} \gamma|-\left(\frac{\tau}{3}-c_{1}\right)|\operatorname{supp} C|\right) \\
& \leqq 2 \sum_{\gamma}\left|\frac{d}{d \mu_{ \pm}} \exp \left(-\psi_{q}^{\prime}\left(\gamma \mid R_{\partial_{e} \gamma}\right)\right)\right| \exp \left(\left(e_{q}+c_{1}\right)|\operatorname{supp} \gamma|\right) .
\end{aligned}
$$

More that either

$$
\frac{d}{d \mu_{ \pm}} \exp \left(-\psi_{q}^{\prime}\left(\gamma \mid R_{\partial_{e^{\gamma}}}\right)\right)=0
$$

or

$$
\frac{d}{d \mu_{ \pm}} \exp \left(-\psi_{q}^{\prime}\left(\gamma \mid R_{\partial_{e} \gamma}\right)=\frac{d}{d \mu} \exp \left(-\psi_{q}\left(\gamma \mid R_{\partial_{e} \gamma}\right)\right) .\right.
$$

Thus, we need only estimate

$$
\begin{aligned}
& \left|\frac{d}{d \mu} \exp \left(-\psi_{q}\left(\gamma \mid R_{\partial_{e}}\right)\right)\right| \\
& \leqq \frac{1}{Z_{q}^{\mathrm{dil}}(\operatorname{Int} \gamma)} \int d R_{V(\gamma)} \chi_{\omega_{\gamma}}\left(R_{\text {supp } \gamma \cup \partial^{\prime} \text { Int } \gamma}\right) \\
& \cdot \exp \left(-H\left(R_{V(\gamma)} \mid R_{\left.\partial_{e}\right\rangle}\right)\right)\left|\frac{d H\left(R_{V(\gamma)} \mid R_{\partial_{e} \gamma}\right)}{d \mu}\right| \\
& +\exp \left(-\psi_{q}\left(\gamma \mid R_{\partial_{e} \gamma}\right)\right) \frac{1}{Z_{q}^{\mathrm{dil}}(\operatorname{Int} \gamma)} \int d R_{\mathrm{Int} \gamma} \chi_{q}\left(R_{\partial^{\prime} \text { Int } \gamma}\right) \\
& \cdot \exp \left(-H\left(R_{\mathrm{Int} \gamma}\right)\right)\left|\frac{d H\left(R_{\mathrm{Int} \gamma}\right)}{d \mu}\right|,
\end{aligned}
$$

where $\partial^{\prime} \operatorname{Int} \gamma$ is the union of all $L$-blocks in Int $\gamma$ which touch $\partial(\operatorname{Int} \gamma)$.

Using assumptions (A.5) we have, for any finite $\Omega \subset Z^{v}$,

$$
\left|\frac{d H\left(R_{\Omega}\right)}{d \mu}\right| \leqq K_{5}(N-1)\left\{(1+2 v) \tilde{\tau}(\beta)|\Omega|+H\left(R_{\Omega}\right)-e_{0}|\Omega|\right\}
$$

and similarly for $H\left(R_{\Omega} \mid R_{\Omega^{\prime}}\right)$. Using the notation $\chi_{\Omega}\left(R_{\Omega}\right)=\prod_{x \in \Omega} \chi_{x}\left(R_{x}\right)$, where $\chi_{x}=1$ or $\chi_{j}$ for some $j=0,1, \ldots, N$, we have integrals of the following form to estimate:

$$
\begin{aligned}
& \int d R_{\Omega} \chi_{\Omega}\left(R_{\Omega}\right) e^{-H\left(R_{\Omega}\right)}\left|\frac{d H\left(R_{\Omega}\right)}{d \mu}\right| \\
& \leqq \int d R_{\Omega} \chi_{\Omega}\left(R_{\Omega}\right) e^{-H\left(R_{\Omega}\right)} K_{5}(N-1)\left\{(1+2 v) \tilde{\tau}(\beta)|\Omega|+H\left(R_{\Omega}\right)-e_{0}|\Omega|\right\} \\
& \leqq K_{5}(2 v+1+\rho) \tilde{\tau}(\beta)|\Omega|(N-1) \int d R_{\Omega} \chi_{\Omega}\left(R_{\Omega}\right) e^{-H\left(R_{\Omega}\right)} \\
& \quad+e^{-e_{0}|\Omega|} K_{5}(N-1) \int_{R_{\Omega} \in D} d R_{\Omega} \exp \left(-\left(H\left(R_{\Omega}\right)-e_{0}|\Omega|\right)\right) \\
& \quad\left(H\left(R_{\Omega}\right)-e_{0}|\Omega|\right) \chi_{\Omega}\left(R_{\Omega}\right),
\end{aligned}
$$


where $D=\left\{R_{\Omega}\left|H\left(R_{\Omega}\right)-e_{0}\right| \Omega|\geqq \rho \tilde{\tau}(\beta)| \Omega \mid\right\}$ and where $\rho>0$ is a constant independent of $\beta$ to be chosen in a moment. Given $\alpha>0$ as in assumption A.2 we now bound the second term on the right-hand side of 6.7) by

$$
\begin{aligned}
& (N-1) K_{5} e^{-e_{0}|\Omega|} \sup _{x \geqq \rho \tilde{\tau}|\Omega|} x e^{-(1-\alpha) x} \int d R_{\Omega} \exp \left(-\alpha\left(H\left(R_{\Omega}\right)-e_{0}|\Omega|\right)\right) \chi_{\Omega} \\
& \leqq(N-1) K_{5} e^{-e_{q}|\Omega|} \exp \left(\frac{K_{1}}{2} \frac{\tilde{\tau}(\beta)}{(2 L)^{v}}|\Omega|\right) \sup _{x \geqq \rho \tilde{\tau}|\Omega|} x e^{-(1-\alpha) x} \cdot\left\{K_{2}+N \frac{\sqrt{2 \pi}}{\eta \alpha}\right\}^{|\Omega|},
\end{aligned}
$$

where we used assumption A.1 and A.2 to bound the integral over $R_{\Omega}$ and (3.7) to bound $e_{0}-e_{q}$. We now choose

$$
\rho=\max \left\{\frac{1}{1-\alpha}, \frac{1}{1-\alpha} \frac{K_{1}}{(2 L)^{v}}\right\}
$$

to conclude that

$$
\begin{aligned}
& \int d R_{\Omega}\left(R_{\Omega}\right) \chi_{\Omega}\left(R_{\Omega}\right) e^{-H\left(R_{\Omega}\right)}\left|\frac{d H\left(R_{\Omega}\right)}{d \mu}\right| \\
& \leqq K_{5}(N-1)(2 v+1+\rho) \tilde{\tau}(\beta)|\Omega| \int d R_{\Omega} \chi_{\Omega}\left(R_{\Omega}\right) e^{-H\left(R_{\Omega}\right)} \\
&+K_{5}(N-1)\left(\frac{N}{\sqrt{\alpha}} e^{-e_{q}-\tau}\right)^{|\Omega|} \rho \tilde{\tau}(\beta)|\Omega|,
\end{aligned}
$$

with $\tau$ as in Lemma 3.1, Eq. (3.4). Combined with (6.5), (6.6) and the bound analogous to (6.8) for $\int d R_{\Omega} \chi_{\Omega} \exp \left(-H\left(R_{\Omega} \mid R_{\Omega^{\prime}}\right)\left|d H\left(R_{\Omega} \mid R_{\Omega^{\prime}}\right) / d \mu\right|\right.$ we obtain the estimate

$$
\begin{aligned}
\frac{1}{|\Lambda|}\left|g_{q}^{(2)}(\mu)\right| \leqq & 4(N-1) K_{5}(2 v+1+\rho) \tilde{\tau}(\beta) \sum_{\gamma} \exp \left(-\left(\frac{\tau}{3}-c_{1}\right)|\operatorname{supp} \gamma|\right) \\
& +2(N-1) K_{5} \rho \tilde{\tau}(\beta) \sum_{\gamma} \frac{\left.\exp \left(e_{q}+c_{1}\right)|\operatorname{supp} \gamma|\right)}{Z_{q}^{\mathrm{dil}}(\operatorname{Int} \gamma)}\left(\frac{N}{\sqrt{\alpha}} e^{-e_{q}-\tau}\right)^{|V(\gamma)|} \\
& +2(N-1) K_{5} \rho \tilde{\tau}(\beta) \sum_{\gamma} \frac{\exp \left(-\left(\frac{\tau}{3}-c_{1}\right)|\operatorname{supp} \gamma|\right)}{Z_{q}^{\mathrm{dil}}\left(\operatorname{Int}_{\gamma}\right)}\left(\frac{N}{\sqrt{\alpha}} e^{-e_{q}-\tau}\right)^{|\operatorname{Int} \gamma|} .
\end{aligned}
$$

Using Theorem $3.3 \mathrm{i}$ to bound $Z_{q}^{\mathrm{dil}}(\operatorname{Int} \gamma)$ and Lemma 3.2 to bound $h_{q}^{\prime}-e_{q}$ we can sum over $\gamma$ and obtain

$$
\frac{1}{|\Lambda|}\left(g_{q}^{(2)}(\mu)\right) \leqq O\left(\tilde{\tau}(\beta) e^{-\tau / 3}\right) .
$$

Combined with (6.4) this proves Lemma 6.2 and hence Theorem 6.1.

\section{Expectation Values}

In this section we construct the infinite volume states $\langle\cdot\rangle_{q}$ corresponding to the dilute partition functions for stable $q$ 's. 
We consider local observables $A$, that is complex valued functions $A=A\left(R_{\operatorname{supp} A}\right)$ depending only on a finite number of variables $R_{x}, x \in \mathscr{A}=\operatorname{supp} A$, for which the norm

$$
\|A\|^{(\alpha)}=\sup _{R_{\mathscr{A}}}\left|A\left(R_{\mathscr{A}}\right)\right| \exp \left(-(1-\alpha) \sum_{x \notin \mathscr{A}}\left[V\left(R_{x}\right)-e_{0}\right]\right)
$$

is finite ( $\alpha<1$ is the constant from assumption A.2). We assume without loss of generality that $\mathscr{A}$ is a connected union of cubes. limit,

In order to construct the expectation value, $\langle A\rangle_{q}$, of $A$ in the infinite volume

$$
\begin{aligned}
\langle A\rangle_{q} & =\lim _{\Lambda \uparrow Z^{v}}\langle A\rangle_{q, \Lambda}^{\mathrm{dil}}, \\
\langle A\rangle_{q, \Lambda}^{\mathrm{dil}} & =Z_{q}^{\mathrm{dil}}(\Lambda)^{-1} \sum_{\omega \in \Omega_{q}(\Lambda)} \int d R_{\Lambda} \chi_{\omega}\left(R_{\Lambda}\right) e^{-H\left(R_{\Lambda}\right)} A,
\end{aligned}
$$

we consider the modification function

$$
Z_{q}^{\mathrm{dil}}(\Lambda, A)=\sum_{\omega \in \Omega_{q}(\Lambda)} \int d R_{\Lambda} \chi_{\omega}\left(R_{\Lambda}\right) e^{-H\left(R_{\Lambda}\right)} A
$$

Our goal is to derive a polymer representation for $Z_{q}^{\mathrm{dil}}(\Lambda, A)$. We always assume that $\Lambda$ is chosen so large that $\operatorname{dist}\left(\mathscr{A}, \Lambda^{c}\right) \geqq 2$.

We proceed as in the derivation of (2.9), the only difference being that we fix not only the external contours of a configuration $\omega \in \Omega_{q}(\Lambda)$, but also all contours $\gamma$ of $\omega$ which are incompatible with $A$, that is all $\gamma$ for which $\operatorname{dist}(V(\gamma), \mathscr{A}) \leqq 1$. We now introduce the notion of an $A$-contour.

Consider a configuration $\omega \in \Omega_{q}(\Lambda)$ and the contours $\gamma_{1}, \ldots, \gamma_{k}$ of $\omega$ which are incompatible with $A$. Let $\gamma_{A}$ be the pair $\left(\operatorname{supp} \gamma_{A}, \omega_{\gamma_{A}}\right)$, where

$$
\operatorname{supp} \gamma_{A}=\mathscr{A} \cup \bigcup_{i=1}^{k} \operatorname{supp} \gamma_{i}
$$

and $\omega_{\gamma_{A}}$ is the restriction of $\omega$ to supp $\gamma_{A}$. We call $\gamma_{A}$ the $A$-contour of $\omega$. Consider a connected component $K$ of $\left(\operatorname{supp} \gamma_{A}\right)^{C}$. Then $\omega$ is constant on $\partial K$ and equals $q$ if $K$ is the infinite component of $\left(\operatorname{supp} \gamma_{A}\right)^{c}$. As before we define $\operatorname{Int}_{m} \gamma_{A}$ to be the union of all finite components, $K$, for which $\omega=m$ on $\partial K$,

$$
\operatorname{Int} \gamma_{A}=\bigcup_{m=1}^{N} \operatorname{Int}_{m} \gamma_{A} \text { and } V\left(\gamma_{A}\right)=\operatorname{Int} \gamma_{A} \cup \operatorname{supp} \gamma_{A} \text {. }
$$

Defining

$$
\exp \left(-\phi\left(\gamma_{A} \mid R_{\gamma_{A}}\right)\right)=A \exp \left(-H\left(R_{\gamma_{A}}\right)\right) \chi_{\omega_{\gamma_{A}}}\left(R_{\gamma_{A}}\right)
$$

and

$$
\begin{aligned}
& \exp \left(-\psi_{q}\left(\gamma_{A} \mid R_{\partial_{e} \gamma_{A}}\right)\right)=\int d R_{\gamma_{A}} \exp \left(-\phi\left(\gamma_{A} \mid R_{\gamma_{A}}\right)\right) \\
& \prod_{\substack{x \in \partial_{A}, y \in e_{e} \gamma_{A} \\
|x-y|=1}} \exp \left(-W\left(R_{x}, R_{y}\right)\right) \prod_{m=1}^{N} \frac{Z_{m}\left(\operatorname{Int}_{m} \gamma_{A}, R_{\partial_{m \gamma_{A}}}\right)}{Z_{q}\left(\operatorname{Int}_{m} \gamma_{A}\right)},
\end{aligned}
$$


one immediately obtains the analog of (2.12), namely

$$
\begin{aligned}
Z_{q}^{\mathrm{dil}}(\Lambda, A)= & \sum_{\gamma_{A}} \sum_{\substack{\in \in \Omega_{q}^{\mathrm{ext}}(\Lambda) \\
C \text { in } \operatorname{Ext}_{A^{\gamma}}}} \int d R_{\Lambda \backslash C} \chi_{q}\left(R_{\Lambda \backslash C_{A}}\right) \\
& \cdot \exp \left(-H\left(R_{\Lambda \backslash C_{A}}\right)\right) \prod_{\gamma \in C_{A}} \exp \left(-\psi_{q}\left(\gamma \mid R_{\partial_{e} \gamma}\right)\right) Z_{q}(\operatorname{Int} \gamma),
\end{aligned}
$$

where the first sum goes over $A$-contours in $\Lambda$ and the second over sets $C=\left\{\gamma_{1}, \ldots, \gamma_{m}\right\}$ of mutually external contours for which each $\gamma_{i}$ lies in $\operatorname{Ext}_{\Lambda} \gamma_{A}=$ $\Lambda \backslash V\left(\gamma_{A}\right) . C_{A}$ is the union of $C$ and $\gamma_{A}: C_{A}=\left\{\gamma_{A}, \gamma_{1}, \ldots, \gamma_{m}\right\}$, and $\Lambda \backslash C_{A} \equiv \Lambda \backslash \operatorname{supp} C_{A}$. Again, (7.8) can be literated giving

$$
\begin{aligned}
Z_{q}^{\mathrm{dil}}(\Lambda, A)= & \sum_{C_{A} \text { in } \Lambda}^{q} \int \exp \left(-\psi_{q}\left(C_{A} \mid R_{\partial_{e} C_{A}}\right)\right) \\
& \cdot \exp \left(-H\left(R_{\Lambda \backslash C_{A}}\right)\right) \chi_{q}\left(R_{\Lambda \backslash C_{A}}\right) d R_{\Lambda \backslash C_{A}},
\end{aligned}
$$

where the sum goes over sets, $C$, of pairwise compatible $q$-contours in $\Lambda$ which contain exactly one $A$-contour. Here compatibility between an ordinary contour $\gamma$ and an $A$-contour, $\gamma_{A}$, is defined by the requirement $\operatorname{dist}\left(V(\gamma), \operatorname{supp} \gamma_{A}\right) \geqq 2$.

We now combine the expansion (7.9) with the high temperature expansion for $Z_{q}^{\text {res }}$. Up to Eq. (4.10) the procedure is exactly the same as in Sect. 4. The only difference arises when we decompose the set supp $C \cup \bar{X} \cup X(\Gamma)$ into polymers where we have to introduce the notion of $A$-polymers. We introduce, for a connected set, $P$, of $L$-blocks, the volume Int $P$ as the union of all finite connected components of $P^{c}$ and the volume $V(P)=P \cup \operatorname{Int} P$. We define: a union $P_{A}$ of $L$-blocks is an $A$-polymer if $P_{A}$ contains the set $\mathscr{A}$ and $\operatorname{dist}(V(P), \mathscr{A}) \leqq 1$ for all connected components $C$ of $P_{A}$. Compatibility between an ordinary polymer $P$ (i.e. a connected union of $L$-blocks) and an $A$-polymer $P_{A}$ is again defined by the requirement

$$
\operatorname{dist}\left(P_{A}, V(P)\right) \geqq 2 \text {. }
$$

We finally introduce the activity $\rho_{A}\left(P_{A}\right)$ by replacing the sum over $C$ in (4.11) by a sum over sets $C_{A}$ containing exactly one $A$-contour, $\gamma_{A}$ (note that $\rho_{A}\left(P_{A}\right)$ is linear in $A$ by our definition of $e^{-\psi_{q}\left(\gamma_{A}, \cdot\right)}$; we also use $\psi_{q}$ instead of $\psi_{q}^{\prime}$ because $q$ is assumed to be stable).

With these definitions we obtain the following analog of (4.72)

$$
Z_{q}^{\mathrm{dil}}(\Lambda, A)=e^{-e_{q}|\Lambda|} Z_{q}^{(0)}(\Lambda) \sum_{\mathbb{P}_{A}} \rho_{A}\left(P_{A}\right) \prod_{i} \rho\left(P_{i}\right),
$$

where the sum goes over sets

$$
\mathbb{P}_{A}=\left(P_{A}, P_{1}, \ldots, P_{k}\right)
$$

of mutually compatible polymers containing exactly one $A$-polymer. We now divide (7.11) by $Z_{q}^{\text {dil }}(\Lambda)$ by the standard algebraic procedure for polymer systems, see e.g. [20]. One obtains

$$
\langle A\rangle_{\Lambda, q}^{\mathrm{dil}}=\sum_{n=0}^{\infty} \sum_{P_{A}, P_{1}, \ldots, P_{n}} \frac{\phi_{c}\left(P_{A}, \ldots, P_{n}\right)}{n !} \rho_{A}\left(P_{A}\right) \prod_{i=1}^{n} \rho\left(P_{i}\right)
$$

where $\phi_{c}$ is the usual combinatoric factor (it is called $a(X)$ in [20]). 
To prove the convergence of this expansion we need the following Lemma, which is the appropriate generalization of Lemma 3.1. $\mathscr{V}^{(1)}$ is the region from Lemma 3.1.

Lemma 7.1. There is a constant $\tilde{c}_{0}$ not depending on $L, \beta$ or $\mu$ such that

$$
\left.\int \mid \exp \left(-\phi_{A} \mid R_{\gamma_{A}}\right)\right) \mid d R_{\gamma_{A}} \leqq \widetilde{K}_{A} \exp \left(-e_{q}\left|\operatorname{supp} \gamma_{A}\right|-\tau\left|\operatorname{supp} \gamma_{A} \backslash \mathscr{K}\right|\right),
$$

provided $\mu \in \mathscr{V}^{(1)}, \tau=\tau(L, \beta)$ is the constant from Lemma 3.1 and

$$
\tilde{K}_{A}=\left(\tilde{c}_{0} e^{e_{q}-e_{0}}\right)^{|\mathscr{A}|}\|A\|^{(\alpha)} \text {. }
$$

Proof. We first extract a factor $\|A\|^{(\alpha)}$ and then proceed as in the proof of Lemma 3.1 to bound

$$
\begin{aligned}
& \int\left|\exp \left(-\phi\left(\gamma_{A} \mid R_{\gamma_{A}}\right)\right)\right| d R_{\gamma_{A}} \leqq\|A\|^{(\alpha)} \exp \left(-e_{0}\left|\operatorname{supp} \gamma_{A}\right|\right) \int \prod_{x \in \mathscr{A}} \exp \left(-\alpha\left(V\left(R_{x}\right)-e_{0}\right)\right) \\
& \cdot \prod_{x \in \operatorname{supp}_{\gamma_{A} \backslash \mathscr{A}}} \exp \left(-\left(V\left(R^{x}\right)-e_{0}\right)\right) \prod_{\langle x y\rangle} \exp \left(-W\left(R_{x}, R_{y}\right)\right) \prod_{x \in \operatorname{supp}_{\gamma_{A}}} \chi_{\omega(x)}\left(R_{x}\right) d R_{x} \\
& \leqq\|A\|^{(\alpha)} \exp \left(-\tilde{\tau}(\mathscr{L}+N)-e_{0}\left|\operatorname{supp} \gamma_{A}\right|\right) c_{0}^{\left|\operatorname{supp}_{A}\right| \cdot \mathscr{A} \mid}\left(c_{0} / \sqrt{\alpha)^{|\mathscr{S}|}},\right.
\end{aligned}
$$

where $N$ and $\mathscr{L}$ are defined as in the proof of Lemma 3.1. Using the fact that all cubes in supp $\gamma_{A} \backslash \mathscr{A}$ are incorrect we obtain $\mathscr{L}+N \geqq(2 L)^{-v}\left|\operatorname{supp} \gamma_{A} \backslash \mathscr{A}\right|$ and hence, again proceeding as in the proof of Lemma 3.1,

$$
\begin{aligned}
& \int\left|\exp \left(-\phi\left(\gamma_{A} \mid R_{\gamma_{A}}\right)\right)\right| d R_{\gamma_{A}} \\
& \quad \leqq\|A\|^{(\alpha)}\left(\frac{c_{0}}{\sqrt{\alpha}} e^{e_{q}-e_{0}}\right)^{|\mathscr{\alpha A |}|} \exp \left(-e_{q}\left|\operatorname{supp} \gamma_{A}\right|\right) \exp \left(-\tau\left|\operatorname{supp} \gamma_{A} \backslash \mathscr{A}\right|\right)
\end{aligned}
$$

provided $\mu \in \mathscr{V}^{(1)}$ (we have used $c_{1} \geqq 1$ without loss of generality). This proves the lemma with $\tilde{c}_{0}=c_{0} / \sqrt{\alpha}$.

Corollary 7.2. For all $b<\infty$ one can choose $L_{0}$ independent of $\beta$ and $L$, and $r_{0}=r_{0}(L)$, $c_{3}=c_{3}(L), \beta_{1}=\beta_{1}(L)$ and $\tau_{1}=\tau_{1}(L)$ not depending on $\beta$, such that

$$
\left|\rho_{A}\left(P_{A}\right)\right| \leqq c_{3}^{|\mathscr{Q}|}\|A\|^{(\alpha)} e^{-b\left|P_{A}\right|},
$$

provided $L \geqq L_{0}, \mu \in \mathscr{V}^{(1)}, \tau=\tau(\beta, L) \geqq \tau_{1}$ and $\beta \geqq \beta_{1}$.

Proof. We fix $\varepsilon, c, \tilde{c}, \tau_{1}=3 \tilde{c}, L \geqq L_{0}$ and $r_{0}$ as in the proof of Lemma 4.1. Assuming without loss of generality that Theorem 3.3 is valid for $\beta \geqq \beta_{1}$ we bound

$$
\begin{aligned}
& \left|\exp \left(-\psi\left(\gamma_{A} \mid R_{\partial_{e} \gamma_{A}}\right)\right)\right| \exp \left(e_{q}\left|\operatorname{supp} \gamma_{A}\right|\right) \\
& \quad \leqq \tilde{K}_{A} \exp \left(-\tau\left|\operatorname{supp} \gamma_{A} \backslash \mathscr{A}\right|+\left(2 c_{1}+1\right)\left|\partial \operatorname{supp} \gamma_{A}\right|\right) \\
& \quad \leqq \widetilde{K}_{A} \exp \left(\left(2 c_{1}+1|\mathscr{A}|-(\tau / 3)\left|\operatorname{supp} \gamma_{A} \backslash \mathscr{A}\right|\right) .\right.
\end{aligned}
$$

If $q$ is stable, $h_{q}^{\prime} \leqq h_{m}^{\prime}$ for all $m$ and, by Lemma 3.2, $e_{q} \leqq e_{m}+2 c_{1}$, which shows that

$$
\tilde{K}_{A} \exp \left(\left(2 c_{1}+1\right)|\mathscr{A}|\right) \leqq\|A\|^{(\alpha)}\left(\tilde{c}_{0} e^{4 c_{1}+1}\right)^{|\mathscr{A}|} .
$$

Combining these bounds with Lemma 5.1 and Lemma 5.2 and proceeding as in 
the proof of Theorem 4.1, one obtains

$$
\begin{aligned}
&\left|\rho_{A}\left(P_{A}\right)\right| \leqq\|A\|^{(\alpha)}\left(\tilde{c}_{0} e^{4 c_{1}+1}\right)^{|\mathscr{A}|} \\
& \sum_{\substack{C_{A}, X \text {,s.t. } \\
\bar{X} \cup \operatorname{supp} C_{A} \subset P_{A}}} \exp \left(-c L^{-v}\left|P_{A}\right|+\left(f_{1}+c L^{-v}\right)\left|\tilde{X} \cup \operatorname{supp} C_{A}\right|\right) \\
& \cdot \exp \left(-\tilde{c}\left|X \cup \operatorname{supp} C_{A} \backslash \mathscr{A}\right|+3^{v} f_{2}\left|X \cup \operatorname{supp} C_{A}\right|\right) .
\end{aligned}
$$

Extracting a factor $e^{\tilde{c}|\mathscr{A}|}$ we can bound the sum as before and obtain the corollary with

$$
c_{3}=\tilde{c}_{0} e^{4 c_{1}+1} e^{\tilde{c}} .
$$

Corollary 7.2, together with Theorem 4.1, immediately imply convergence of the cluster expansion (7.12), and hence the following

Theorem 7.3. There are constants $\varepsilon>0, K<\infty$ and $\beta^{*}<\infty$, such that the following statements are true for $\beta \geqq \beta^{*}$ and $\mu \in \mathscr{V}^{(1)}$.

i) For stable boundary conditions $q$, and any observable $A$ with $\|A\|^{(\alpha)}<\infty$, the thermodynamic limit

$$
\langle A\rangle_{q}=\lim _{\Lambda \rightarrow Z^{v}}\langle A\rangle_{q, \Lambda}^{\mathrm{dil}}
$$

exists, is translation invariant and shows exponential clustering.

ii) Let $\langle\cdot\rangle_{q}^{\text {res }}$ be the infinite volume state corresponding to $Z_{q}^{\text {res }}$. Then, for stable boundary conditions $q$,

$$
\left|\langle A\rangle_{q}-\langle A\rangle_{q}^{\text {res }}\right| \leqq K^{|\operatorname{supp} A|}\|A\|^{(\alpha)} e^{-\varepsilon \tilde{\tau}(\beta)},
$$

where $\tilde{\tau}(\beta)$ is the constant from assumption A.2.

Proof. i) is standard and ii) is proven by comparing the cluster expansion for $\langle A\rangle_{q}$ and $\langle A\rangle_{q}^{\text {res }}$. Since the activities of these two expansions differ only by terms containing at least one contour, we may extract a factor $e^{-\tilde{\varepsilon} \tau} \leqq e^{-\tilde{\tau}(\beta)}$; the remaining sums are bounded as before giving the factor $K^{|\operatorname{supp} A|}\|A\|^{(\alpha)}$.

The following theorem is proven in the same way as Theorem $7.3 \mathrm{ii}) .\langle\cdot\rangle_{q}^{(0)}$ denotes the infinite volume state of the Gaussian with covariance $C_{q}=\left(m_{q}^{2}-\kappa_{q} \Delta\right)^{-1}$ and mean $R_{q} . \delta(\beta)$ is the constant from assumption A.1.

Theorem 7.4. For a polynominally bounded function $A(\cdot)$ denote by $A_{q}$ the shifted function, $A_{q}(R)=A_{q}\left(R-R_{q}\right)$. Then, for $\beta>\beta^{*}$,

$$
\left|\left\langle A_{q}\right\rangle_{q}^{\mathrm{res}}-\left\langle A_{q}\right\rangle_{q}^{(0)}\right| \leqq K_{A} \delta(\beta)^{\varepsilon},
$$

where $K_{A}$ depends on $A$, but not on $\beta$, and $\varepsilon>0$ is independent of $\beta$ and $A$.

Remark. One may have observed that the definition of $\langle\cdot\rangle_{q}$ in Sect. 1 differs from that given here. Since $Z_{q}(\Lambda)$ and $Z_{q}^{\mathrm{dil}}(\Lambda)$ and hence also $\langle\cdot\rangle_{q, \Lambda}$ and $\langle\cdot\rangle_{q, \Lambda}^{\mathrm{dil}}$ differ only by contours touching $\partial \Lambda$, one may easily check that

$$
\lim _{\Lambda \rightarrow Z^{v}}\langle A\rangle_{q, \Lambda}=\lim _{\Lambda \rightarrow Z^{v}}\langle A\rangle_{q, \Lambda}^{\text {dil }}
$$

if $q$ is stable, $\beta \geqq \beta^{*}$ and $\|A\|^{(\alpha)}<\infty$. 
Acknowledgements. We thank Jürg Fröhlich for steering us towards this problem and its solution as well as for many discussions and suggestions, particularly concerning the mean field expansions of Sect. 4. We also thank John Imbrie for several discussions. Finally, we are both indebted to Krystof Gawedzki and the hospitality of the I.H.E.S. where this work was begun.

\section{References}

1. Borgs, C., Waxler, R.: First order phase transitions in unbounded spin systems II. Completeness of the phase diagram. Commun. Math. Phys. (in press)

2. Glimm, J., Jaffe, A., Spencer, T.: A convergent expansion about mean field theory. Ann. Phys. 101, 610-630 and 631-669 (1976)

3. Brydges, D.: A rigorous approach to Debye screening in dilute classical coulomb systems. Commun. Math. Phys. 58, 313-350 (1978)

4. Brydges, D., Federbush, P.: Debye screening. Commun. Math. Phys. 73, 197-246 (1980)

5. Balaban, T., Gawedzki, K.: A low temperature expansion for the pseudoscalar Yukawa model of quantum fields in two spaces time dimensions. Ann. Inst. Henri Poincaré 36, 271-400 (1982)

6. Imbrie, J.: Phase diagrams and cluster expansions for low temperature $P(\phi)_{2}$ Models. Commun. Math. Phys. 82, 261-304 and 305-343 (1981)

7. Pirogov, S., Sinai, Ya.: Phase transitions of the first kind for small perturbations of the Ising model. Funct. Anal. Appl. 8, 21-25 (1974)

8. Pirogov, S., Sinai, Ya.: Phase diagrams of classical lattice spin systems. Theor. Math. Phys. 25, 1185-1192 (1975) and Theor. Math. Phys. 26, 39-49 (1976)

9. Sinai, Ya.: Theory of phase transitions: rigorous results. Oxford: Pergamon Press 1982

10. Borgs, C., Imbrie, J.: A unified approach to phase diagrams in field theory and statistical mechanics. Commun. Math. Phys. 123, 305 (1989)

11. Zahradnik, M.: An alternative version of Pirogov-Sinai theory. Commun. Math. Phys. 93, 559-581 (1984)

12. Bricmont, J., Kuroda, K., Lebowitz, J. L.: First order phase transitions in lattice and continuous systems: extension of Pirogov-Sinai theory. Commun. Math. Phys. 101, 501-538 (1985)

13. Dobrushin, R. L., Zahradnik, M.: Phase diagrams for continuous-spin models: an extension of the Pirogov-Sinai theory. In: Dobrushin, R. L. (ed.). Mathematical problems of Statistical Mechanics and Dynamics. Dordrecht: Reidel 1986

14. Zahradnik, M.: Low temperature continuous spin gibbs states on a lattice and the interfaces between them-a Pirogov-Sinai type approach. In: Dorlas, T., Hugenholtz, N. M., Winnik, M. (eds.). Statistical mechanics and field theory: mathematical aspects. (Groningen, 1985). Lecture Notes in Physics. Berlin, Heidelberg, New York: Springer 1986

15. Malyshev, V. A.: Cluster expansions in lattice models of statistical physics and the quantum theory of fields. Russ. Math. Surv. 35, 1-62 (1980)

16. Dobrushin, R. L.: A new approach to the analysis of gibbs perturbations of gaussian fields. Preprint 1988

17. Borgs, C., Fröhlich, J., Waxler, R.: The phase structure of the large $n$ Lattice Higgs Model, ETH-Preprint TH 89/11, to appear in Nucl. Phys. B.

18. Balaban, T., Brydges, B., Imbrie, J., Jaffe, A.: The mass Gap for Higgs' models on a unit lattice. Ann. Phys. 158, 281-319 (1984)

19. Brydges, D.: A short course on cluster expansions. In: Osterwalder, K., Stora, R. (ed.). Critical phenomena, random systems, gauge theories. (Les Houches 1984). Amsterdam: North Holland 1986

20. Seiler, E.: Gauge theories as a problem of constructive quantum field theory and statistical mechanics. Lecture Notes in Physics vol. 159. Berlin, Heidelberg, New York: Springer 1982 